\title{
The Parkes quarter-Jansky flat-spectrum sample
}

\section{Sample selection and source identifications ${ }^{\star}$}

\author{
C. A. Jackson ${ }^{1}$, J. V. Wall ${ }^{2}$, P. A. Shaver ${ }^{3}$, K. I. Kellermann ${ }^{4}$, I. M. Hook ${ }^{2}$, and M. R. S. Hawkins ${ }^{5}$ \\ 1 Research School of Astronomy \& Astrophysics, The Australian National University, Mount Stromlo Observatory, \\ Canberra, Australia \\ 2 Department of Astrophysics, University of Oxford, Nuclear and Astrophysics Laboratory, Keble Road, \\ Oxford OX1 3RH, UK \\ 3 European Southern Observatory, Karl-Schwarzschild-Str. 2, 85748 Garching bei München, Germany \\ 4 National Radio Astronomy Observatory, Edgemont Road, Charlottesville, VA 22903-2475, USA \\ 5 Royal Observatory, Blackford Hill, Edinburgh EH3 9HJ, UK
}

Received 7 November 2001 / Accepted 22 January 2002

\begin{abstract}
We present a new sample of quarter-Jansky flat-spectrum radio sources selected to search for highredshift quasars and to study the evolution of the flat-spectrum quasar population. The sample comprises 878 radio sources selected from the Parkes catalogues with spectral indices $\alpha_{2.7}^{5} \mathrm{GHz}$ covers all right ascensions and the declination range from $-80^{\circ} 0$ to +2.5 , excluding low galactic latitudes $(|b|<$ $10^{\circ}$ ) and the Magellanic Cloud regions. We have obtained improved radio source positions, firstly to reconfirm the majority of the existing identifications, and secondly, using digitized sky-survey data and deep B, Gunn- $i$ and Gunn- $z$ CCD-imaging, to find optical identifications for 223 previously-unidentified sources. We present the final catalogue of 878 flat-spectrum sources: 827 are compact radio sources identified with galaxies, quasars and BL Lac objects, 38 have either extended radio structure or are identified with Galactic objects (PN, HII or non-compact radio source), 4 are obscured by Galactic stars, and 9 (1 per cent of the total sample) remain unidentified.
\end{abstract}

Key words. catalogues - radio continuum: galaxies - BL Lac objects: general - galaxies: general - quasars: general

\section{Introduction}

This is the first paper in a series of three describing the results of a program to search for high-redshift quasars and study the evolution of the flat-spectrum quasar population.

This paper (Paper I) sets out the sample, discussing its selection and the identification and reconfirmation programme to determine the optical counterparts to the radio sources. Paper II (Hook et al. in preparation) presents new spectroscopic observations and redshift determinations. Paper III (Wall et al. in preparation) considers quasar space distribution, the luminosity function and its epoch dependence. An earlier version of this sample was used by

Send offprint requests to: C. A. Jackson,

e-mail: cjackson@mso.anu.edu.au

* Full Appendices A-D are only available in electronic form at http://www.edpsciences.org
Shaver et al. (1996) to study the evolution of the quasar space density at redshifts greater than 3 .

We selected our radio source sample from the machinereadable version of the composite Parkes radio source catalogue (PKSCAT90: Wright \& Otrupcek 1990). This catalogue comprises radio and optical data collected for 8264 radio sources for the sky south of $+27^{\circ}$, excluding the Galactic Plane and Magellanic Cloud regions. The catalogue was compiled from major radio surveys at $2.7 \mathrm{GHz}$ undertaken by John Bolton and colleagues over the years 1969 to 1979 (Bolton et al. 1979 and references therein).

Samples extracted from PKSCAT90 contain sourceidentification information compiled over the last 30 years or more in numerous collaborations, resulting in several complete or near-complete samples, e.g. the 2-Jy sample (Wall \& Peacock 1985), the Parkes Selected Regions (Downes et al. 1986; Dunlop et al. 1989, 1991) and the Parkes half-Jansky sample (Drinkwater et al. 1997). 
Our goal was to obtain a spectroscopic sample of flatspectrum radio-selected quasars, free of optical selection effects and complete at high redshifts. Starting with a sample selected from PKSCAT90 and the identifications therein we therefore had to ensure that we (i) identified the remaining PKSCAT90 sources and (ii) obtained redshifts for all candidate quasars in the sample, with priority given to those north of $-40^{\circ}$ declination.

The results of our selection, identification and confirmation programme are described in this paper as follows. Section 2 gives the selection criteria for the sample and some characteristics of the identified and empty field sources. In Sect. 3 we describe the procedure for identifying the PKSCAT90 empty-field sources and in Sect. 4 we analyse the reliability of the complete sample. In Sect. 5 we present the full catalogue and in Sect. 6 we summarise the characteristics of the final sample.

\section{Radio source selection}

The sample of flat-spectrum radio sources was selected from PKSCAT90 using the following criteria -

1. $-80.0<\delta(\mathrm{B} 1950)<+2.5$, all right ascensions;

2. $\alpha_{2.7 \mathrm{GHz}}^{5.0 \mathrm{GHz}}>-0.4$, where $S \propto \nu^{\alpha}$;

3. Galactic latitude $|b|>10^{\circ}$.

Sources lying within the Magellanic Cloud regions (B1950 $\mathrm{RA}$, Dec $=0430 \mathrm{hrs}$ to $0630 \mathrm{hrs},-64^{\circ}$ to $-75^{\circ}$ for the LMC and $00 \mathrm{hrs}$ to $02 \mathrm{hrs},-69^{\circ}$ to $-76^{\circ}$ for the SMC) were excluded from our sample. We also checked the $2.7 \mathrm{GHz}$ and $5 \mathrm{GHz}$ flux densities extracted from PKSCAT90 against the original survey papers listed in Table 2. A number of sources have more recent flux density measurements recorded by PKSCAT90; we replaced these with the original survey flux densities for consistency. In addition, we find 10 sources in PKSCAT90 which are not in the original survey papers (Table 1): 3 of these sources lie in regions not covered by the original Parkes surveys. The other 7 sources are within the original survey areas listed in Table 2 but are not in the original survey papers.

A cut-off value of -0.4 for the spectral index was applied to minimize the number of extended or non-coredominated radio sources in the sample. However, such sources still contaminate the sample, due to one or more of the following reasons:

1. Flux density uncertainties: the original Parkes survey flux densities at $2.7 \mathrm{GHz}$ and $5 \mathrm{GHz}$ have uncertainties of $\left((0.02)^{2}+(0.03 S)^{2}\right)^{0.5}$. Therefore there will be objects with $\alpha_{2.7 \mathrm{GHz}}^{5 \mathrm{GHz}}<-0.4$ in the sample.

A few nearby sources have a different type of fluxdensity error, due to diffuse lobe emission that has been poorly mapped and errors in one or both flux densities can result in $\alpha_{2.7 \mathrm{GHz}}^{5 \mathrm{GHz}}<-0.4$. PKS B0123-016 $(3 \mathrm{C} 40)$ is a case in point.
Table 1. PKSCAT90 sources not in original Parkes Survey papers.

\begin{tabular}{ll}
\hline \hline Source & Region $^{(1)}$ \\
\hline PKS B0522-030 & A \\
PKS B0528-454 & $\mathrm{J}$ \\
PKS B0549-459 & $\mathrm{J}$ \\
PKS B1131-115 & $\mathrm{B}$ \\
PKS B1150-723 & $\mathrm{K}$ \\
PKS B1159-515 & $\mathrm{H}$ \\
PKS B1718-649 & $\mathrm{X}$ \\
PKS B1730-130 & X \\
PKS B1801+010 & X \\
PKS B2111+015 & A \\
\hline
\end{tabular}

Notes: (1) The original Parkes survey region (see Table 2).

2. Confusion: the Parkes beam sizes of 8 arcmin at $2.7 \mathrm{GHz}$ and 4 arcmin at $5 \mathrm{GHz}$ result in occasional instances of source confusion.

3. Source variability: there are two effects here. The first is due to the time interval between the $2.7 \mathrm{GHz}$ and $5 \mathrm{GHz}$ flux density measurements; a number of steepspectrum sources are included in our sample whilst a number flat-spectrum sources are missed as the source varied. The second effect is that neither flux density determination measured the mean source flux density, with the result that a number of sources are missed or included depending on their activity at the time of the survey. Drinkwater et al. (1997) analysed the magnitude of these effects for the 0.5-Jy Parkes survey sample which has a steeper spectral-index selection $\left(\alpha_{2.7}^{5.0 \mathrm{GHz}}>-0.5\right)$ and concluded that 1$)$ the time lag between the surveys includes about 3 per cent steepspectrum sources and misses a similar proportion of true flat-spectrum sources, and 2) the difference between the Parkes survey flux densities and mean flux densities results in $\sim 11$ per cent of the sample having mean flux densities below any survey area's flux density limit, whilst $\sim 8$ per cent of sources are missed. The latter bias is inherent in any single-epoch flux-limited sample.

To assess the magnitude of these effects we have compared 694 sources common to our sample and NVSS (Condon et al. 1998). We find that the vast majority -663 out of 694 (96 per cent) - are either compact (unresolved by NVSS, major axis $<15$ arcsec) or are extended sources with an obvious dominant radio core so that unambiguous identification is possible. Thus we conclude that the incidence of non-core dominated, steep-spectrum sources in our complete sample is less than 5 per cent.

Figure 1 shows the distribution of the 878 quarterJansky sample sources within the Parkes survey regions. 
Table 2. The Parkes Survey regions.

\begin{tabular}{|c|c|c|c|c|c|}
\hline \multirow[b]{2}{*}{ Area } & \multirow[b]{2}{*}{ Dec range } & \multicolumn{4}{|c|}{$S_{\lim , 2.7 \mathrm{GHz}}$} \\
\hline & & $\mathrm{RA}$ range & / Jy & Reference & $\mathrm{N}^{(1)}$ \\
\hline A & +2.5 to $-4^{\circ}$ & $7^{\mathrm{h}} 20$ to $17^{\mathrm{h}} 50,19^{\mathrm{h}} 40$ to $6^{\mathrm{h}}$ & $0.25^{(2)}$ & Wall et al. (1971) & $147(+2)$ \\
\hline $\mathrm{B}$ & $-4^{\circ}$ to $-15^{\circ}$ & $10^{\mathrm{h}}$ to $15^{\mathrm{h}}$ & 0.25 & Bolton et al. (1979) & $56(+1)$ \\
\hline $\mathrm{C}$ & $-15^{\circ}$ to $-30^{\circ}$ & $10^{\mathrm{h}}$ to $15^{\mathrm{h}}$ & 0.25 & Savage et al. (1977) & 65 \\
\hline $\mathrm{D}$ & $-4^{\circ}$ to $-30^{\circ}$ & $22^{\mathrm{h}}$ to $5^{\mathrm{h}}$ & 0.25 & Wall et al. (1976) & 157 \\
\hline \multirow[t]{2}{*}{$\mathrm{E}$} & $-4^{\circ}$ to $-30^{\circ}$ & $5^{\mathrm{h}}$ to $6^{\mathrm{h}} 30,8^{\mathrm{h}}$ to $10^{\mathrm{h}}$ & & & \\
\hline & & $15^{\mathrm{h}}$ to $17^{\mathrm{h}}, 19^{\mathrm{h}}$ to $22^{\mathrm{h}}$ & 0.6 & Bolton et al. (1975) & 64 \\
\hline $\mathrm{F}$ & $-18^{\circ} .8 \pm 2^{\circ} .6$ & $22^{\mathrm{h}} \pm 2.6$ & 0.10 & Wall et al. (1971) & 9 \\
\hline G & $-30^{\circ}$ to $-35^{\circ}$ & $9^{\mathrm{h}}$ to $16^{\mathrm{h}} 30,18^{\mathrm{h}} 30$ to $7^{\mathrm{h}} 15$ & 0.2 & Shimmins \& Bolton (1974) & 91 \\
\hline $\mathrm{H}$ & $-33^{\circ}$ to $-75^{\circ}$ & $3^{\mathrm{h}}, 11^{\mathrm{h}}, 19^{\mathrm{h}}, 23^{\mathrm{h}}$ & 0.25 & Shimmins (1971) & $66(+1)$ \\
\hline I & $-35^{\circ}$ to $-45^{\circ}$ & $10^{\mathrm{h}}$ to $15^{\mathrm{h}}, 19^{\mathrm{h}}$ to $7^{\mathrm{h}}$ & 0.25 & Bolton \& Shimmins (1973) & 79 \\
\hline \multirow[t]{2}{*}{$\mathrm{J}$} & $-45^{\circ}$ to $-65^{\circ}$ & $0^{\mathrm{h}}$ to $3^{\mathrm{h}}, 4^{\mathrm{h}}$ to $8^{\mathrm{h}} 30$ & & & \\
\hline & & $17^{\mathrm{h}} 30$ to $19^{\mathrm{h}}, 20^{\mathrm{h}}$ to $23^{\mathrm{h}}$ & h 0.5 & Wall et al. (1975) & $74(+2)$ \\
\hline $\mathrm{K}$ & $-65^{\circ}$ to $-75^{\circ}$ & $0^{\mathrm{h}}$ to $24^{\mathrm{h}}$ & 0.25 & Bolton \& Butler (1975) & $32(+1)$ \\
\hline $\mathrm{L}$ & $-75^{\circ}$ to $-80^{\circ}$ & $0^{\mathrm{h}}$ to $24^{\mathrm{h}}$ & 0.2 & Shimmins \& Bolton (1972) & 28 \\
\hline $\mathrm{X}$ & all other areas & not covered by original Parkes surveys & 0.25 & PKSCAT90 & 3 \\
\hline
\end{tabular}

Notes: (1) Number of sources each region contributes to our sample. The number in parenthesis is the number of sources which are in this region but not in the referenced paper (see Table 1). (2) Region A includes 21 sources lying in 5 regions, each of 40 square degrees with $S_{2.7 \mathrm{GHz}}=0.1$ Jy as shown in Fig. 1.

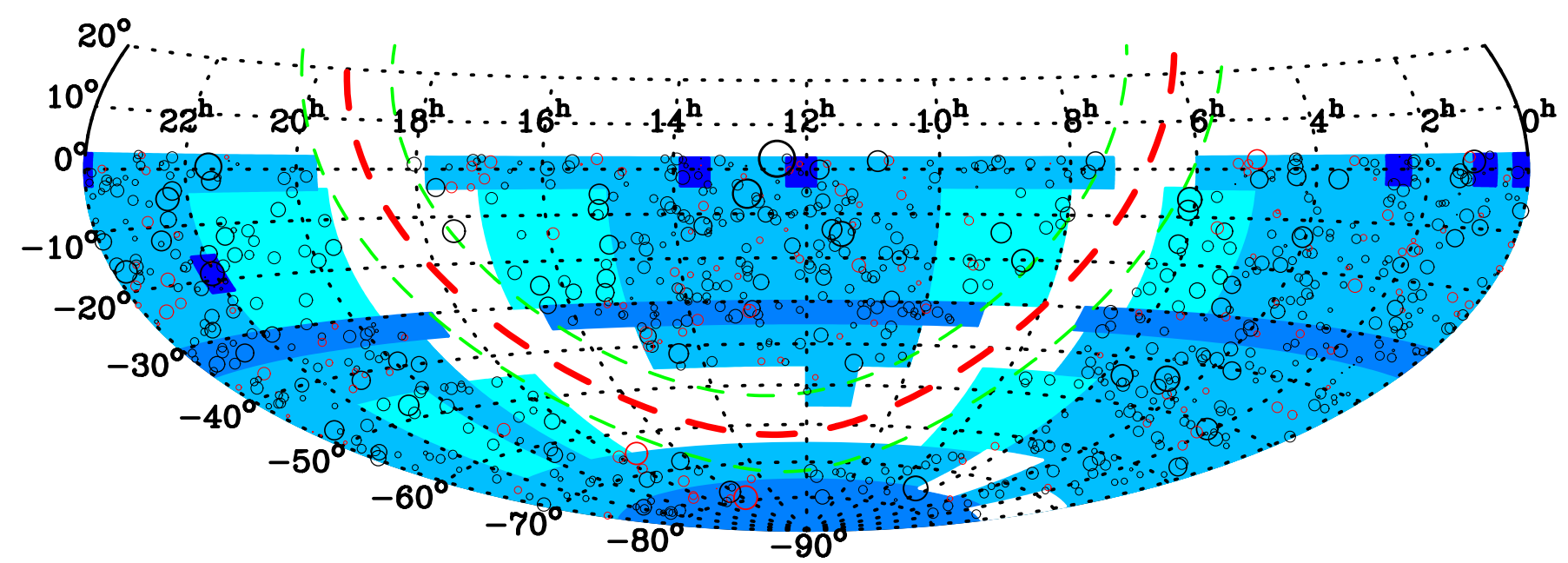

Fig. 1. Sky distribution of the Parkes quarter-Jansky flat-spectrum sources within the Parkes survey regions. Radio sources identified with galaxies and stellar objects are shown as circles with the size of the circle being proportional to the $2.7 \mathrm{GHz}$ flux density. Parkes $2.7 \mathrm{GHz}$ survey limits are shown in shades of grey, from dark to light, equating to $S_{2.7 \mathrm{GHz}}$ of $0.10,0.20,0.25$ and $0.60 \mathrm{Jy}$. Sources in the Magellanic Cloud regions and near the Galactic equator $\left(|b|<10^{\circ}\right)$ are excluded from the sample.

Of these, 645 have an optical identification given in PKSCAT90, with the majority, 535 (82 per cent), with stellar objects (quasars or BL Lacs). Moreover, it is the brighter radio sources which are identified, leaving the majority of the 247 unidentified (Parkes empty-field) sources with $2.7 \mathrm{GHz}$ flux densities below 0.6 Jy (Fig. 2). In contrast, the distribution of spectral indices between identified and unidentified sources is unbiased (Fig. 3). 


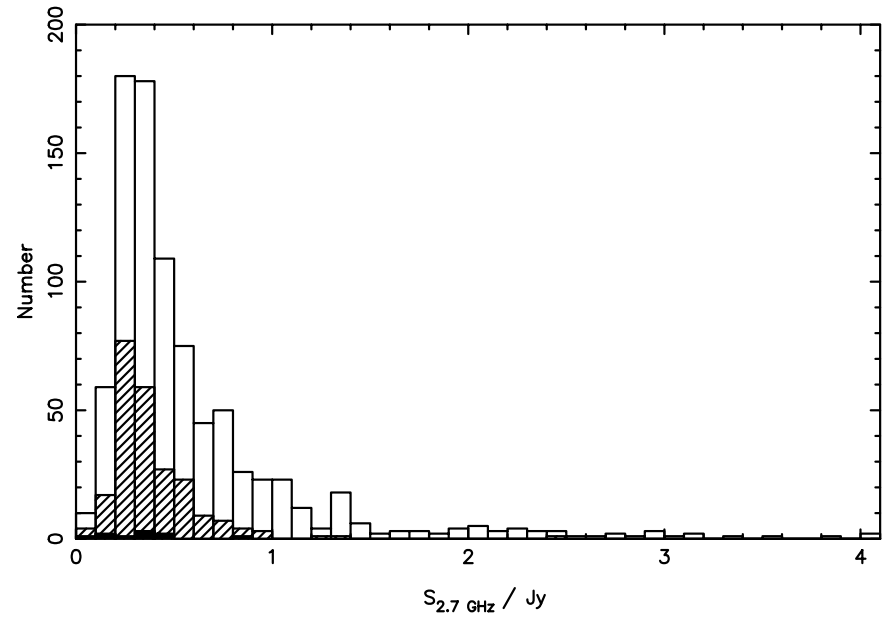

Fig. 2. $2.7 \mathrm{GHz}$ flux density distributions for the quarterJansky sample (open histogram). Flux densities for our 223 new identifications shown hatched and the 9 remaining empty fields shown solid fill.

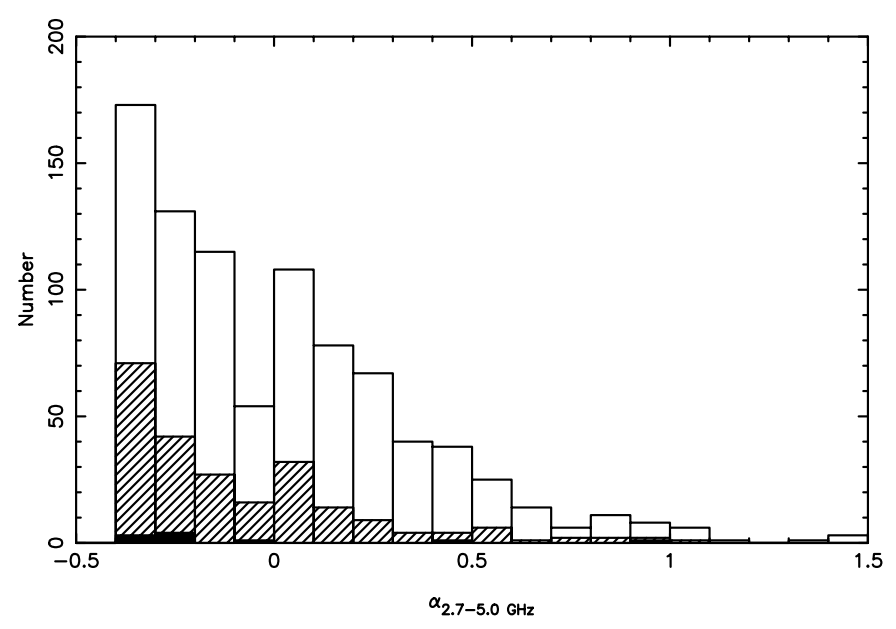

Fig. 3. 2.7-5 GHz spectral index distributions for the quarterJansky sample $\left(S \propto \nu^{\alpha}\right)$ (open histogram). Spectral indices for our 223 new identifications shown hatched and the 9 remaining empty fields shown solid fill.

\section{The identification procedure}

Identification of the PKSCAT90 empty-field sources was carried out as follows:

1. Accurate radio positions to better than 1 arcsec were obtained for all PKSCAT90 empty-field sources (Sect. 3.1).

2. The COSMOS digitized sky survey database was interrogated at the new radio positions to find candidate source identifications (Sect. 3.2).

3. Where no candidate was found from the COSMOS database, deep CCD images were taken through blue and red filters (Sect. 3.3).

4. We then obtained low-dispersion spectra for all stellar identifications, as these are potential high-redshift $(z>$ 3) quasars (Paper II).

5. A random subset of COSMOS candidate identifications was also imaged to assess the incidence of blending of faint objects by COSMOS which would otherwise result in mis-identifications.

6. The reliability of the total sample was then assessed (Sect. 5): (i) for the new identifications; (ii) for the existing PKSCAT90 identifications.

\subsection{Radio observations}

Refined radio positions are necessary to identify the empty field sources as the original Parkes radio positions are accurate only to $\sim 10-15$ arcsec. We obtained these data for 72 of the empty field sources via our own observations with the Very Large Array (VLA) and Australia Telescope Compact Array (ATCA).

For 63 sources south of declination $-30^{\circ}$ the ATCA was used in the " $6 \mathrm{D}$ " configuration to obtain accurate radio positions. On 27 September 1993 observations were made at 4.80 and $8.64 \mathrm{GHz}$ with orthogonal linear polarisations and a bandwidth of $128 \mathrm{MHz}$. The synthesised beam at $4.80 \mathrm{GHz}$ was $2 \operatorname{arcsec} F W H M$ (east-west). Each source was observed in typically three 45 -s cuts widely spaced in hour angle, and these one-dimensional cuts were later combined to determine the source position. Secondary position calibrators were interspersed regularly throughout these observations. The flux density scale was based on the ATCA primary calibrator, PKS 1934-638.

In 1994 and 1995 we used the VLA to determine accurate positions of 29 previously unidentified sources. Most of these are are declinations north of $-30^{\circ}$ but a few are as far south as $-41^{\circ}$. The observations were made at $8.4 \mathrm{GHz}$ with the VLA in its "A" or "B" configuration with corresponding resolutions of 0.4 to 1.2 arcsec. Each programme source was covered with a single "snapshot" scan typically of about four minutes duration, and each group of snapshots was preceded and followed by scans on a phase calibrator whose rms position uncertainty is not more than 0.1 arcsec in each coordinate. The flux density scale was based on an assumed value of $5.2 \mathrm{Jy}$ for 3C 286 .

The VLA data were edited and calibrated using the AIPS software package.

The remainder of the new radio positions were obtained from both previously published and unpublished results. The origin of each position is shown in the final catalogue (Appendix A). All radio positions used in the identification process are accurate to better than 1 arcsec in each coordinate. Throughout, B1950 positions were used, and where necessary the STARLINK SLALIB and $\mathrm{COCO}$ routines have been used to convert from other epochs and equinoxes.

Our VLA and ATCA observations revealed four sources with extended radio structures and no well-defined position or obvious optical counterpart. A further five Parkes sources were undetected at the VLA. For these, we found no evidence of any compact structure stronger than 0.5 mJy within 1 arcmin of the Parkes position. They are coded as "NCS" (non-compact radio sources) in the final catalogue (Appendix A) and no optical identification was attempted. 


\subsection{Optical identifications using sky survey data}

The plates of the ESO/SERC Southern Sky Survey were taken with a IIIa-J emulsion and a GG395 filter to give the photographic blue passband $B_{\mathrm{J}}(3950 \AA-5400 \AA)$, with a limiting magnitude $\left(m_{B_{\mathrm{J}}}\right)$ of approximately 22.5 . These plates were digitized with the COSMOS platemeasuring machine at the Royal Observatory Edinburgh (Yentis et al. 1992). The COSMOS database was used to generate finding charts two arcmin-square centered on the radio position. Details of the COSMOS object nearest to the radio position were recorded.

There is an error in the stored coordinates in the COSMOS database arising from the precession calculations used to construct the database at ROE. The effect of this is that the positions stored in the COSMOS database are not J2000 but are close to B2000 (see Drinkwater et al. 1995 for a full discussion). As a result the "B1950" positions produced from COSMOS are also incorrect. As the COSMOS positions have been constructed using the "FK4" catalogue there is no mean systematic offset in the COSMOS positions from the (FK4) SAO catalogue. However, there is a systematic offset between COSMOS and the newer (FK5) Positions and Proper Motions (PPM) catalogue (Roeser et al. 1994). The PPM catalogue is more accurate than the SAO catalogue in that the FK5 system is closer to an inertial reference frame. For the level of astrometric accuracy required by this project, the COSMOS coordinates must be corrected by reference to an FK5 catalogue.

In order to correct the COSMOS "B1950" positions to true B1950 positions for each field, $(x, y)$ positions for approximately 12 of the faintest PPM stars around each radio position were measured on the ESO R survey plate using the PDS plate-measuring machine at the Royal Greenwich Observatory. The ESO R survey was chosen as the images are smaller, less saturated than deeper surveys, and hence a more accurate determination of the star centres was possible. Where the ESO R survey was not available the UKST IIIa-J survey was used. The $(x, y)$ coordinates were transformed to (RA, Dec) coordinates using a 6-coefficient fit to Schmidt distortion geometry using STARLIB ASTROM. Each COSMOS "B1950" position was then corrected from the average of the offsets.

With the corrections applied, we initially took the nearest COSMOS-database object to the radio position as the optical counterpart. A Gaussian fitted to the radio - optical offsets revealed an rms scatter of $\sim 1.12 \operatorname{arcsec}$ in each coordinate. We therefore took a maximum offset of $3.6 \operatorname{arcsec}(\sim 3 \sigma)$ in each coordinate as the identification criterion.

Objects which were obviously extended or were found to have narrow-line spectra (Paper II) were classified as galaxies, the remainder as stellar objects. If a candidate was close to the sky survey plate limit $\left(b_{\mathrm{J}} \sim 22\right)$, in the absence of other data for the object we conservatively classified it as stellar.
Table 3. ESO 3.6-m imaging observations.

\begin{tabular}{lc}
\hline \hline Dates & $\begin{array}{c}\text { Number of } \\
\text { nights used }\end{array}$ \\
\hline 20-23 April 1993 & 3 \\
10-14 November 1993 & 4 \\
10-12 May 1994 & 2 \\
5-8 October 1994 & 3 \\
26-29 April 1995 & 2 \\
23-25 October 1995 & 3 \\
\hline
\end{tabular}

Using the COSMOS database, we found identifications for 136 of the PKSCAT90 empty-field sources, comprising 107 stellar objects and 29 galaxies. The galaxy identifications were confirmed via visual inspection of the sky survey plates, as often faint objects in close proximity are merged by the COSMOS digitization process and then erroneously recorded in the database as a single object (usually a "galaxy").

\section{3. $C C D$ Imaging}

To identify the 87 sources which had no candidate identification from the COSMOS database we conducted a programme of deep CCD-imaging using the ESO Faint Object Spectrograph and Camera (EFOSC1) at the Cassegrain focus of the ESO 3.6 m-telescope. Six observing sessions were undertaken at the telescope, as shown in Table 3.

ESO filters \#552 (B), \#614 (Gunn- $i$ ) and \#615 (Gunn- $z$ ) were used for imaging on EFOSC1, using photometric standards from Stobie et al. (1985) and Landolt (1992). Typical integration times were 5 min in $B$ and 10 min in Gunn- $i$ and Gunn- $z$ filters.

The raw FITS-format CCD images obtained with EFOSC1 were reduced using the Image Reduction and Analysis Facility (IRAF) version 2.10, developed at NOAO. The radio source position $(x, y)$ was aligned onto the CCD frames via the STARLINK ASTROM routine using the positions of approximately 12 faint stellar objects on the CCD frame. The corrected COSMOS B1950 positions of these stellar objects were transformed using a 6 -coefficient fit which was accepted when the rms residual reduced to $\lesssim 0.3$ arcsecs. In many cases we obtained more than one exposure per radio source so that an $(x, y)$ solution was required for each frame. All frames were then manually examined for candidate objects.

For a source to be accepted as the optical counterpart we adopted the same $3 \sigma$ criterion as for the COSMOS identifications. For the accepted identifications we find that the CCD-based identifications have a wider Gaussian spread of radio - optical offsets as shown in Fig. 4 and Table 4 with the CCD identifications generally associated with fainter optical sources (Fig. 5). 


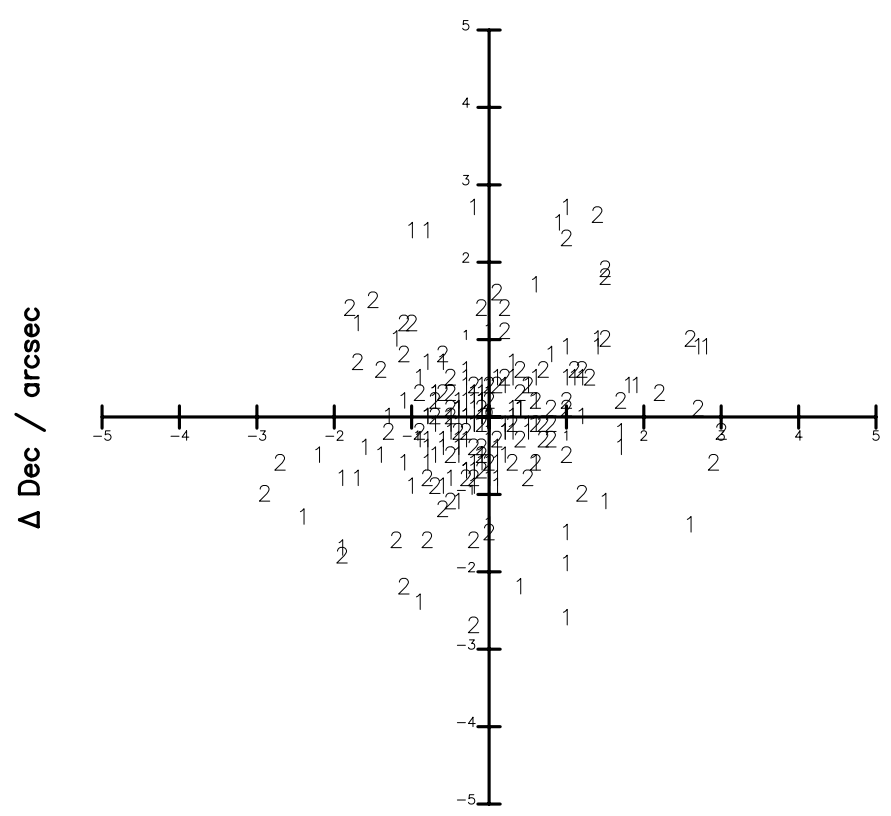

$\Delta \mathrm{RA} / \operatorname{arcsec}$

Fig. 4. Position offsets in RA and dec for the 223 new identifications. Identifications from COSMOS are shown as "1" and those from CCD imaging as "2".

Table 4. Radio-optical position offsets.

\begin{tabular}{lrrrrr}
\hline \hline & & $\langle\Delta \mathrm{RA}\rangle$ & $\sigma_{\mathrm{RA}}$ & $\langle\Delta \mathrm{Dec}\rangle$ & $\sigma_{\mathrm{Dec}}$ \\
Identification & $N$ & $/ \operatorname{arcsec}$ & $\operatorname{arcsec}$ & $\operatorname{arcsec}$ & $\operatorname{arcsec}$ \\
\hline COSMOS-survey & 136 & -0.06 & 0.89 & -0.04 & 0.85 \\
CCD images & 87 & 0.07 & 1.12 & 0.03 & 0.96 \\
\hline
\end{tabular}

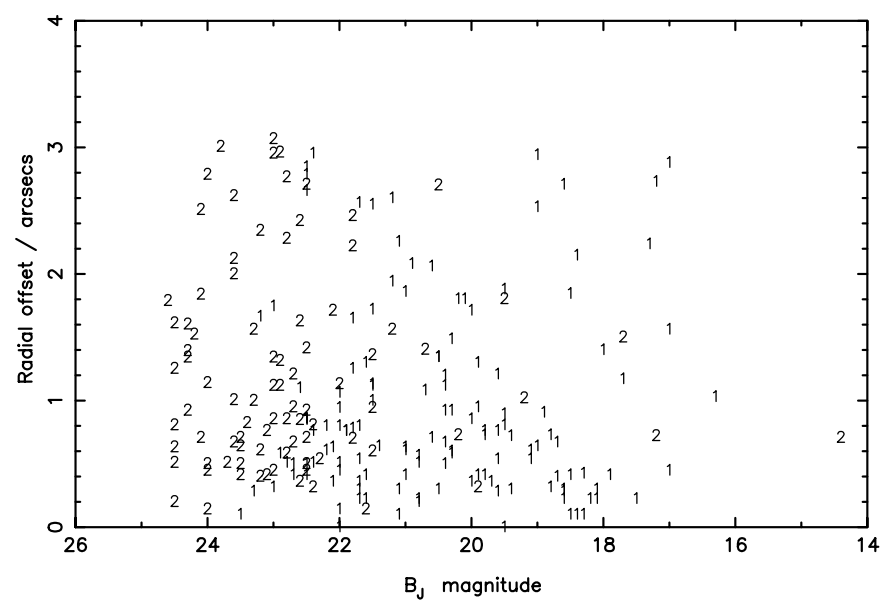

Fig. 5. Position offsets (radial) for the 223 new identifications. Identifications from COSMOS are shown as "1" and those from CCD imaging as "2".

Our CCD-imaging programme resulted in identifications for 87 sources. These identifications comprise 37 stellar objects and 50 galaxies. In Appendix D we show the CCD images, each 2 arcmin square, centred on the new identifications. The classification of type (stellar object/galaxy) was based on observed morphology. For 31 sources (23 stellar objects and 8 galaxies) we have confirmed their classifications via low-resolution spectroscopy (Paper II).

\section{Reliability of the new identifications}

There is the possibility that erroneous identifications have been made with objects lying close to the line-of-sight of the radio source. The uncertainty in the radio and optical positions and the density of objects on the survey plate or CCD exposure both determine the chance coincidence of a non-radio source being identified in error. Contamination due to foreground objects is fairly obvious where the object is a galactic star but not so obvious when the object is a faint galaxy.

The background faint-galaxy density was determined by examination of the deepest CCD exposures in each of the filters. The count of the galaxy density at the CCD imaging limit $\left(m_{\mathrm{B}}=24.5\right)$ is found to be 12 faint galaxies per square arcmin in B, 13 per square arcmin in Gunn$i\left(m_{\mathrm{Gunn}-i}=23.5\right)$ and 9 per square arcmin in Gunn- $z$ $\left(m_{\text {Gunn-z }}=25.0\right)$. There is a 10 per cent chance of a faint galaxy falling within the search area for any one identification. In the majority of identifications the candidate is a far brighter object so that the chance of it being an erroneous identification is remote. However, there are 38 faint galaxy identifications $\left(m_{\mathrm{B}} \geq 23\right)$, so that 4 are potential spurious identifications. We find that the rms radio minus optical offsets for these 38 faint galaxy identifications is larger (RA, dec: 1.19, 0.92 arcsec) than those for the brighter identifications (RA, Dec: 0.93, 0.89 arcsec).

The new identifications can therefore be considered as being reliable, although this is inevitably a function of magnitude. Additional confidence in the identifications is provided by the histogram of $m_{\mathrm{B}}$ magnitudes for the new identifications (Fig. 6). The decline in new identifications occurs around $b_{\mathrm{J}}=23$, i.e. at about one magnitude brighter than the limiting magnitude of our CCD-images.

\section{The sample}

\subsection{Sample catalogue}

The catalogue of the quarter-Jansky sample is presented in Appendix A. Finding charts, images and additional notes on individual sources are given in Appendices B, C and D.

The identification classes in our catalogue, column 8, are as follows. $\mathrm{G}=$ galaxy, and in this we have subsumed the PKSCAT90 classes "DB", "E" and "Sey", defining sub-categories of galaxies (double, elliptical and Seyferts respectively). $\mathrm{Q}=$ quasar, confirmed spectroscopically or via UVX measurement, while $\mathrm{Q}^{*}=$ quasar by virtue of coincidence between the position of a stellar object and an 


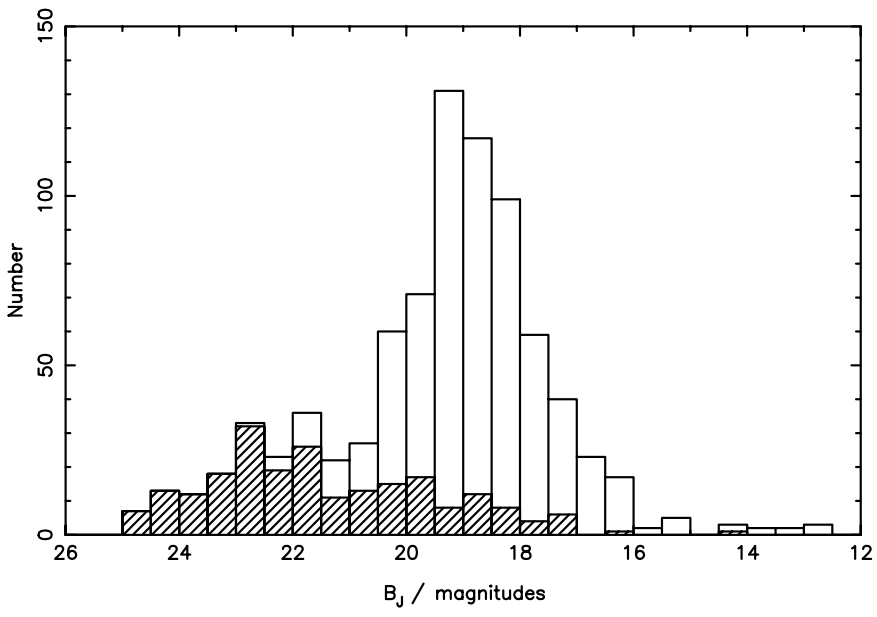

Fig. 6. Distribution of optical $b_{\mathrm{J}}$ magnitudes for all galaxy, quasar and BL Lac sources in the quarter-Jansky sample, with our new identifications shown hatched.

accurate radio position, and Q? is an unconfirmed quasar, a stellar object coincident with a Parkes survey radio position.

\subsection{Catalogue reliability}

In the course of constructing the final catalogue and subsequent to the analysis of Shaver et al. (1996) we have reassessed the data contained in the entire catalogue. Our primary aim is to ensure that we have not overlooked any $z>5$ candidate quasars in the sample, particularly due to errors in PKSCAT90. For the 233 new identifications discussed in Sect. 3 we are confident in that there are no undiscovered high-redshift quasars.

We use digitized material from the UKST IIIa-J and UK SES R surveys along with the best-available radio positions to check every one of the 878 sources in our sample. The overlays of the radio position on the sky survey data is shown in Appendix C.

We find 10 radio sources for which we cannot confirm the identification (see Table 5). Of these 10, one source, PKS B0141-060 lies within an area already covered by the Sydney University Molonglo Sky Survey (SUMSS, Bock et al. 1999) at $843 \mathrm{MHz}$ : This is clearly a complex, extended source so this is classified as a non-compact source (NCS).

Of the remaining 9 sources, 3 have radio positions catalogued by NVSS (Condon et al. 1998): PKS B0225-065, PKS B0415-029 and PKS B0522-030. There is no more recent radio survey data from which to confirm the validity of the other 7 sources. As an interesting point of note, three of the remaining unidentifiable PKSCAT90 sources in Table 5 do not appear in the original Parkes survey papers (Table 2): That such a significant fraction of the non-Parkes survey sources cannot be identified suggests that they are either highly variable or that their existence is questionable.
Table 5. PKSCAT90 previously-identified sources deidentified as a result of arcsec-radio positions.

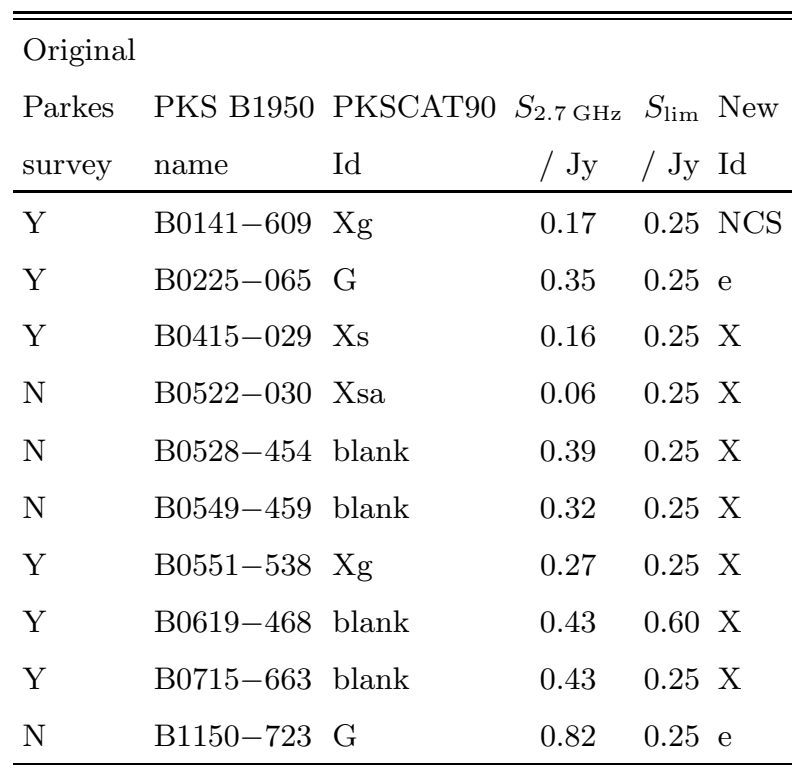

Considering the PKSCAT90 identifications we find that two sources, PKS B0225-065 and PKS B1150-723 are identified as galaxies. However, as we find no evidence to support this, we code these sources to "e" for an elimated PKSCAT90 identification. Another source, PKS B0551-538 is classified as "Xg" in PKSCAT90, indicating at least one galaxy within the Parkes radio position error box. We code this as an empty-field source (X). Two sources are PKSCAT90 empty-field sources ("Xs" and "Xsa") and were overlooked in our identification campaign and remain empty fields (X). The remaining four PKSCAT90-blank coded sources also are coded as emptyfields $(\mathrm{X})$.

For the PKSCAT90 confirmed identifications we have assessed the stellar identifications as follows: $\mathrm{Q}=$ quasar, confirmed spectroscopically or via UVX measurement, while $\mathrm{Q}^{*}=$ quasar by virtue of coincidence between the position of a stellar object and an accurate radio position, and $\mathrm{Q}$ ? is an unconfirmed quasar, a stellar object coincident with a Parkes survey $(\sim 10-15$ arcsec $)$ radio position.

Furthermore, we have checked all redshifts in the table by searching the original references. Numerous errors in the PKSCAT90 listing were found. Full references for the redshifts appear in Appendix A, and Appendix B gives full details of updates to PKSCAT90 data.

\section{Sample characteristics}

\subsection{Optical magnitude distribution}

The distribution of $b_{\mathrm{J}}$ magnitudes for the sample is shown in Fig. 6; our new identifications (inevitably) lie at the faint end of the distribution and show that the total 


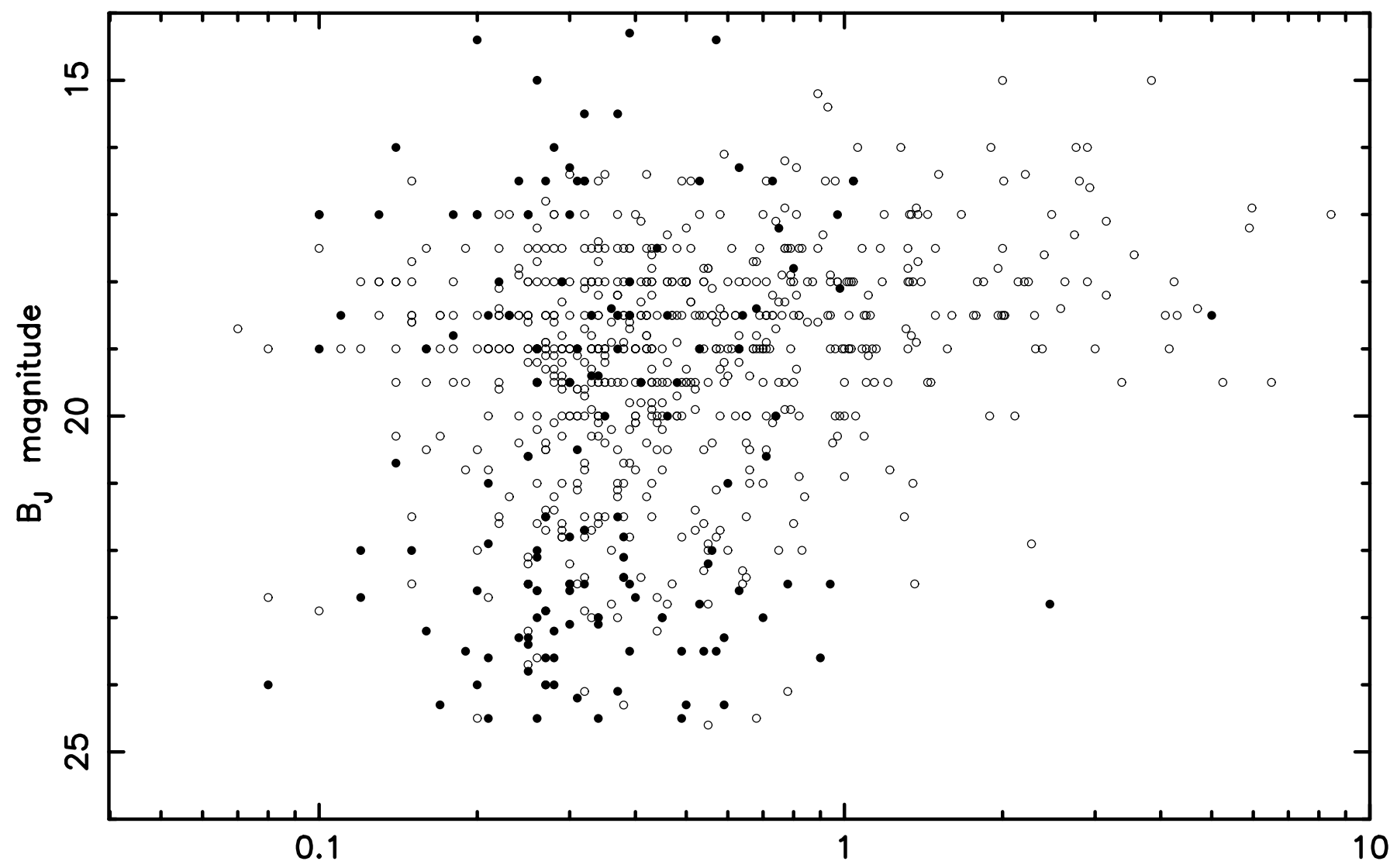

\section{7-GHz flux density / Jy}

Fig. 7. Optical $b_{\mathrm{J}}$ magnitudes vs. radio flux densities $\left(b_{\mathrm{J}}\right.$ vs. $S_{2.7 \mathrm{GHz}}$ ) for 150 galaxies $(\bullet)$ and 677 stellar objects $($ o, quasars and BL Lacs) from the quarter-Jansky radio sample.

distribution is mildy bimodal with a main peak at $b_{\mathrm{J}}=$ $19.5 \mathrm{mag}$ and a subpeak near $b_{\mathrm{J}}=22.5 \mathrm{mag}$.

Figure 7 , a plot of the sample in $b_{\mathrm{J}}$ magnitude vs. $S_{2.7 \mathrm{GHz}}$ with indication of object type, shows that galaxies dominate at faint flux densities and faint $b_{\mathrm{J}}$ magnitudes. Considering identification-fraction as a function of $b_{\mathrm{J}}$ magnitude (Fig. 8) we see that quasars dominate between $b_{\mathrm{J}}=16$ to 22 , and that the spectroscopicallyconfirmed BL Lac objects comprise 5 to 10 per cent of the sample at all $b_{\mathrm{J}}$ magnitudes. However, this latter result should be treated as a lower limit only, as we do not have complete spectroscopic measurements for all stellar sources.

\subsection{Source types as a function of $2.7 \mathrm{GHz}$ flux density}

As a function of flux density (Fig. 9), the galaxies are significant (i) at bright flux densities (the cores of active nearby ellipticals) and (ii) towards the faint flux densities $\left(S_{2.7 \mathrm{GHz}} \sim 0.2 \mathrm{Jy}\right)$. The increase in the galaxy fraction at fainter flux densities has also been observed in other radio samples (Falco et al. 1998) and probably comprises peaked-spectrum or compact symmetric radio galaxies.

The set of histograms of Fig. 10 shows how the $b_{\mathrm{J}}$ magnitude distribution changes with decreasing $2.7 \mathrm{GHz}$ flux density. The brightest radio sources have a peaked distribution around $b_{\mathrm{J}}=18.5$; there is no hint of bimodality as there is little intrusion of the flat-spectrum population of faint galaxies. As radio flux density decreases and the flatspectrum galaxies appear, the distribution takes on the bimodal shape, which continues below $S_{2.7 \mathrm{GHz}}=0.25 \mathrm{Jy}$. As first noted by Bolton (1970), the median of the distribution shifts to fainter magnitudes as flux density decreases, an effect seen in subsequent studies (Browne \& Wright 1985; Drinkwater et al. 1997).

\subsection{Redshift distribution}

The redshift distribution is shown in Fig. 11. There is some dependence on flux density in that the distribution for the fainter sources of the sample - those with flux densities in the range $0.25 \leq S_{2.7 \mathrm{GHz}}<0.5 \mathrm{Jy}-$ is flatter and biased 


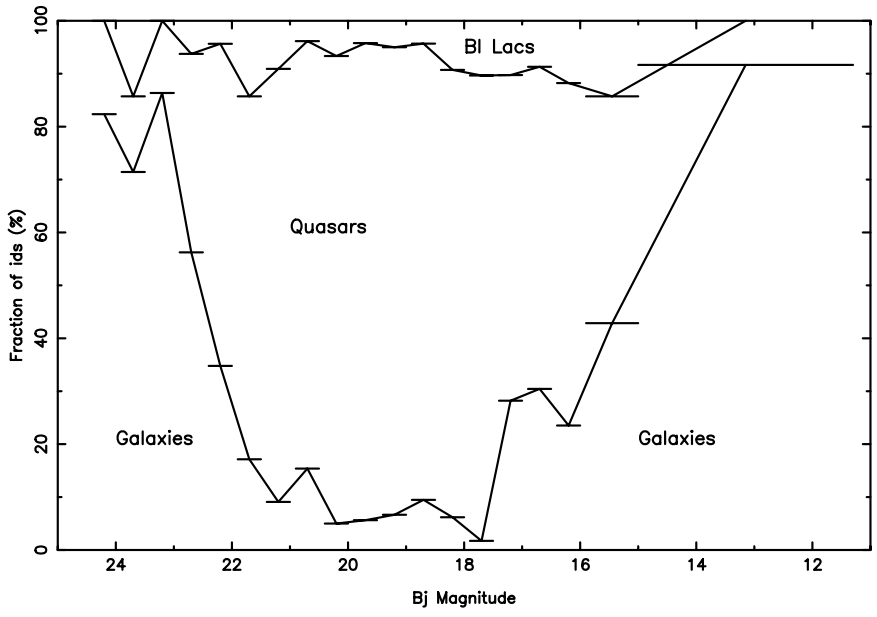

Fig. 8. Proportions of identified source types by optical $b_{\mathrm{J}}$ magnitude in the quarter-Jansky sample.

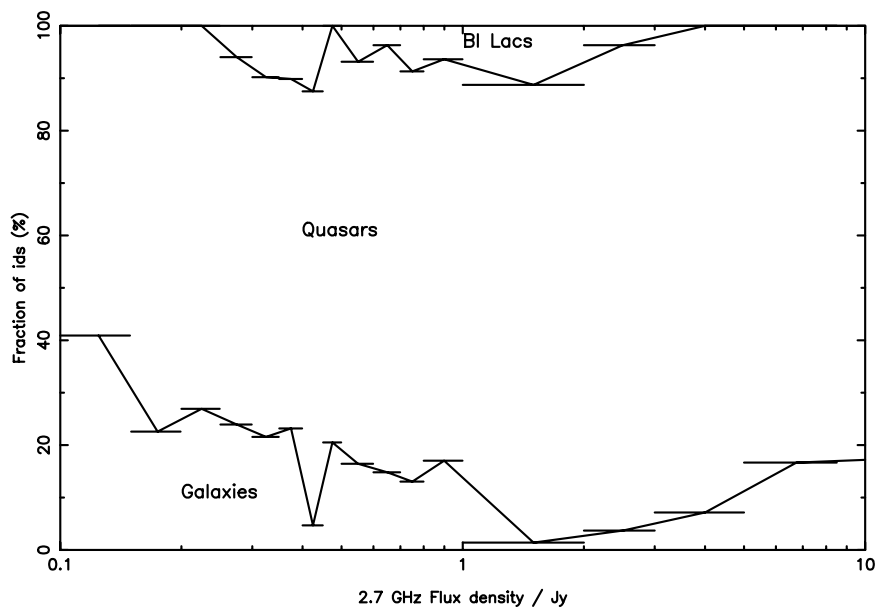

Fig. 9. Proportions of the identified source types by $S_{2.7 \mathrm{GHz}}$ in the quarter-Jansky sample.

to significantly higher redshifts than that for all sources with $S_{2.7 \mathrm{GHz}} \geq 0.25 \mathrm{Jy}$.

\subsection{Spectral index distributions}

The distributions of spectral indices for galaxies and stellar objects (quasars and BL Lacs) are compared in Fig. 12. A K-S test reveals that there is only a 2.3 per cent probability that the two distributions are drawn from the same population. The galaxy identifications have generally steeper spectra; their distribution is suggestive simply of an extended tail to the classical (low-frequency survey) distribution with its peak around $\sim-0.8$. The quasar plus BL Lac distribution gives indication of the bimodality seen so clearly in samples selected at frequencies above $2.7 \mathrm{GHz}$. In these sources the flat-spectrum peak is identified with beamed emission.
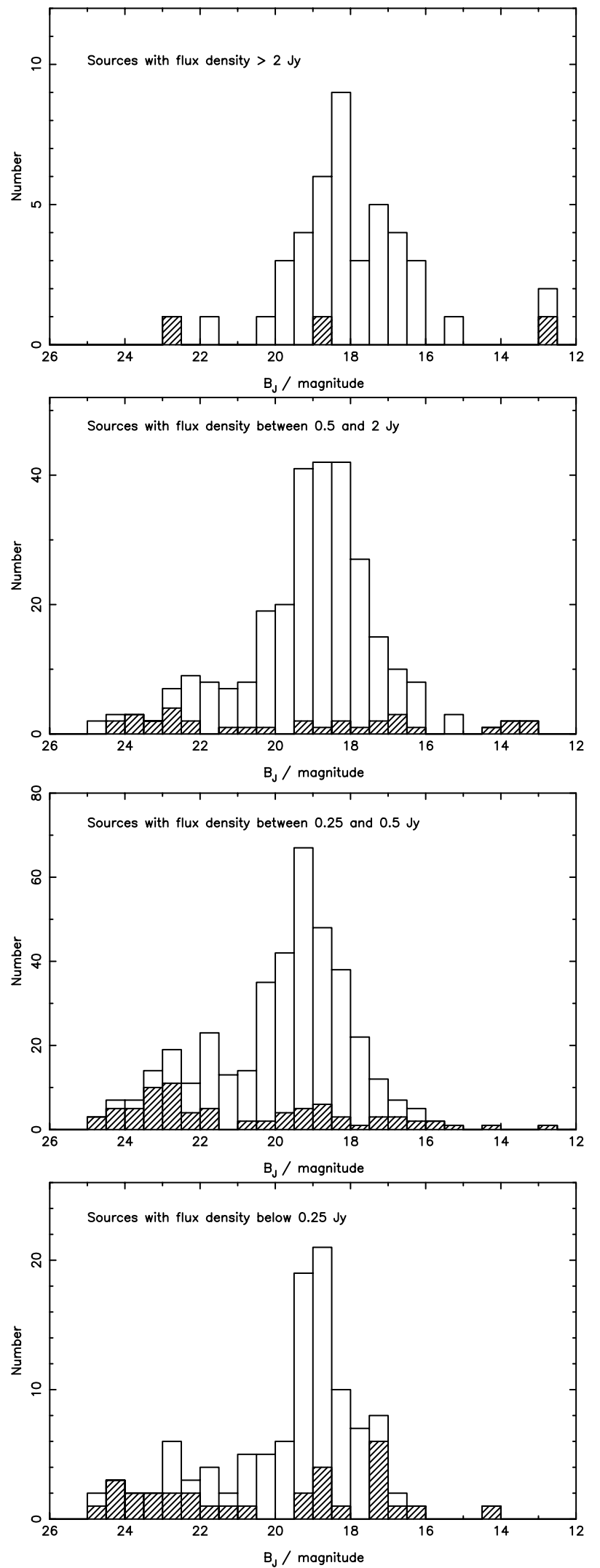

Fig. 10. Optical $b_{\mathrm{J}}$ magnitude distributions a) for sources with $S_{2.7 \mathrm{GHz}} \geq 2.0 \mathrm{Jy}$ b) for sources with $S_{2.7 \mathrm{GHz}} \geq 0.50 \mathrm{Jy}$ c) for sources with $S_{2.7 \mathrm{GHz}} \geq 0.25 \mathrm{Jy}$ and $\mathbf{d}$ ) for sources with $S_{2.7 \mathrm{GHz}}<0.25 \mathrm{Jy}$. Legend: hatched regions indicate galaxy identifications, open histogram regions quasar plus BL Lac identifications. 

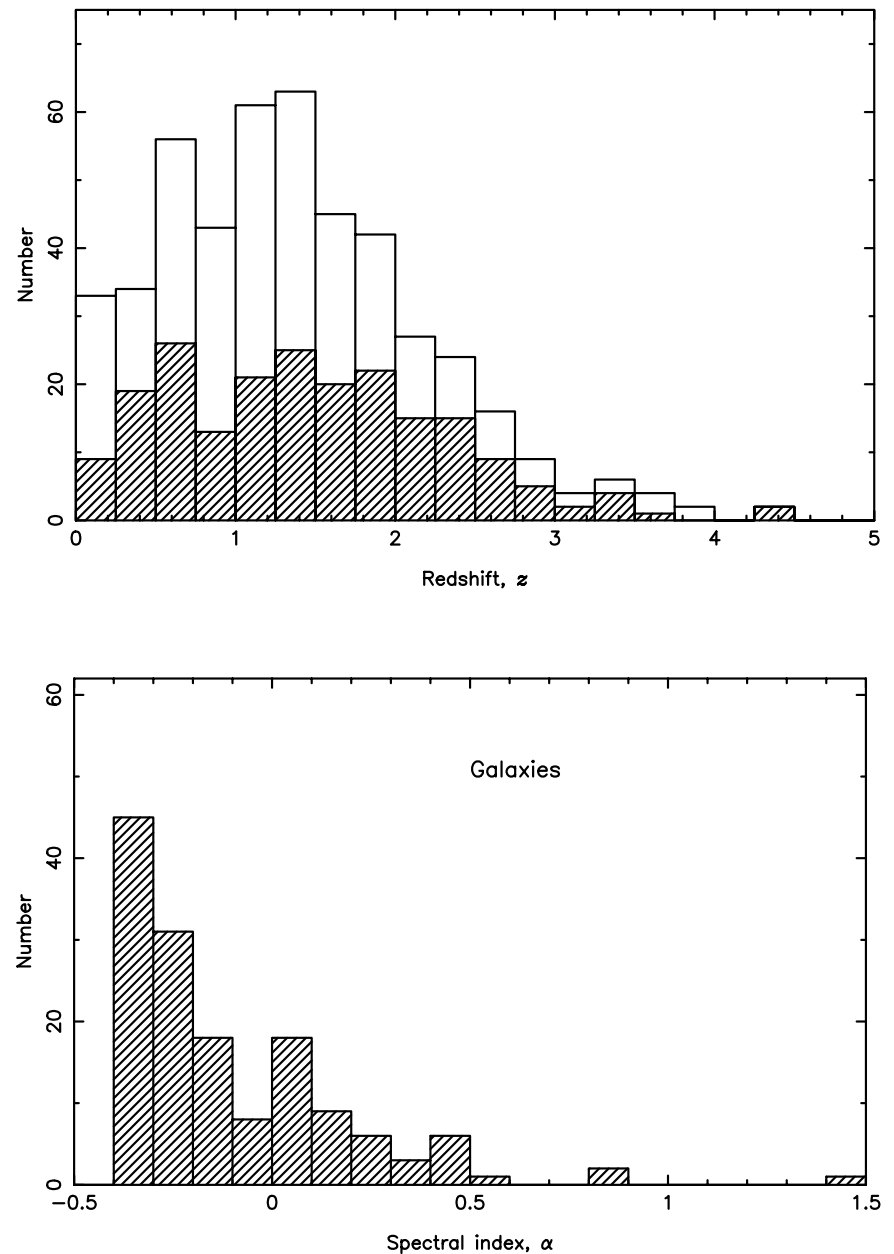

Fig. 11. Redshift distribution for the quarter-Jansky sample sources $S_{2.7 \mathrm{GHz}} \geq 0.25 \mathrm{Jy}$ (unfilled histogram). For comparison sources with $0.25 \leq S_{2.7} \mathrm{GHz}<0.50 \mathrm{Jy}$ are shown hatched.

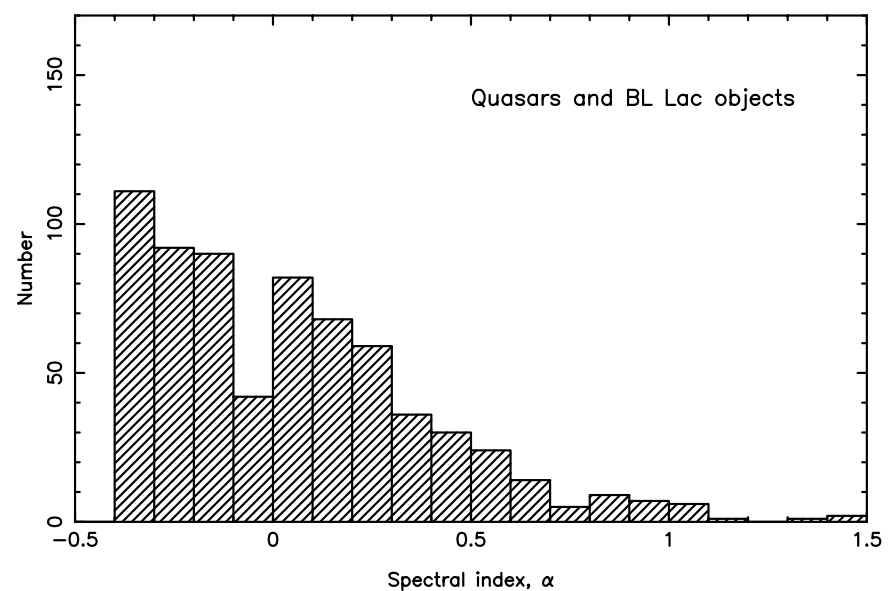

Fig. 12. Spectral index distributions $\left(\alpha_{2.7 \mathrm{GHz}}^{5 \mathrm{GHz}}\right)$ by identified source types in the quarter-Jansky sample sources. Left - galaxies; right - quasars and BL Lac objects.

\section{Summary}

In a sample of 878 flat-spectrum sources selected from PKSCAT90, observations to obtain accurate radio positions and optical imaging data have resulted in optical counterparts for 223 previously unidentified sources. The sample catalogue presented here contains 827 compact radio sources identified with galaxies, quasars and BL Lac objects, 38 sources either resolved or identified with Galactic objects, and 4 sources whose fields are optically contaminated by bright stars. Just 9 sources (1 per cent) remain unidentified. From this total sample, complete flux-density limited samples can be selected; for example for sources north of $-40^{\circ}$ declination in regions where the survey limit is $\geq 0.25$ Jy there are secure identifications for 418 out of 419 sources (99.8 per cent).

Further to our comprehensive checking of the existing PKSCAT90 data and our new identifications, we conclude that

- it contains no $z>5$ objects, and
- the increasing contribution of galaxies in the sample below about $0.2 \mathrm{Jy}$ is real and these are probably peaked-spectrum or compact symmetric sources.

The sample highlights why completion of optical identifications is difficult even for flat-spectrum compact samples: at faint flux-density levels $\left(S_{2.7 \mathrm{GHz}}<0.3 \mathrm{Jy}\right)$ a significant fraction of the host objects are faint red galaxies.

Acknowledgements. We thank Dick Hunstead, Alok Patnaik, John Reynolds, Ann Savage and Niven Tasker for providing radio positions in advance of publication. We also thank Dave McConnell and Bob Sault for assistance with the ATCA observations and analysis, Benoit Germain for his work with the optical data reduction and John Pilkington for carrying out the PDS plate measurements. This work is based on data collected at the European Southern Observatory, the Australia Telescope National Facility and the National Radio Astronomy Observatory. NRAO is operated by Associated Universities Inc. under cooperative agreement with the National Science Foundation. 


\section{Appendix A: The complete catalogue of the Parkes quarter-Jansky sample}

Catalogue column entries:

1. PKSCAT90 B1950 source name.

2. and 3. PKSCAT90 radio source position (B1950).

4. and 5. The best radio source position (B1950).

6. The origin of the radio position given in Cols. 4 and 5:

A ATCA observation for this project

C ATCA calibrator (ftp.atnf.csiro.au/pub/atnfdocs/guides/at.cat)

$\mathrm{H}$ R Hunstead, private communication

N NVSS catalogue (Condon et al. 1998)

$\mathrm{P} \quad$ A Patnaik, private communication

R J Reynolds, private communication

$\mathrm{S}$ A Savage, private communication

$\mathrm{T}$ N Tasker, private communication

$\mathrm{V}$ VLA observation for this project

W White (1992)

7. Origin of identification:

1: Reconfirmed PKSCAT90 identification based on the following:

1a: Sky survey data and the best radio position available as shown in the table. No offsets (radio - optical) have been determined for these sources.

1b: COSMOS sky survey and the best radio position available as shown in the table. Identification offsets (radio - optical) have been determined for these sources.

1c: CCD imaging and the best radio position available as shown in the table. Identification offsets (radio optical) have been determined for these sources.

2: A new identification by this project using the COSMOS database.

3: A new identification by this project using CCD-imaging.

8. Identification:

Q: confirmed quasar by spectroscopy or UVX photometry

$\mathrm{Q}^{*}$ : stellar object based on positional coincidence with accurate (post-PKSCAT90) radio position, although no spectrum or UVX confirmation.

Q?: PKS radio position coincident with stellar object. No spectroscopy/UVX confirmation.

BL: spectroscopically-confirmed BL Lac object.

G: Galaxy with morphological or spectroscopic confirmation.

G?: probably a galaxy based on optical colour or morphology.

$\mathrm{X}$ : no identification, was previously a PKSCAT90 empty-field source.

e: the best radio invalidates the PKSCAT90 identification in that there is no optical source at the best radio position.

Ob: radio position obscured by foreground star.

NCS: Non-compact radio source. The source is either a steep-spectrum or extended radio source. Refer to individual notes for full details (Appendix B).

PN: Planetary nebula.

HII: HII region.

9. and 10. Radio - optical offset of candidate identification (given for identification classes 1c, 2 and 3 in Col. 7).

11. $B$ or $B_{\mathrm{J}}$ magnitude. The origin of these magnitudes can be determined from the entry in Col. 7 as follows: for " 1 " identifications, the magnitude is from PKSCAT90 and could be $B$ or $B_{\mathrm{J}}$, for " 2 " identifications the magnitudes is from COSMOS and is a $B_{\mathrm{J}}$ magnitude, for " 3 " identifications the magnitude is from our CCD-imaging and is a B magnitude.

Magnitudes prefixed with: are either (i) estimated (ii) untraceable in the literature although given in PKSCAT90 or (iii) too close to the DSS plate limit to be reliable.

12. and 13. Gunn- $i$ and Gunn- $z$ (instrumental) magnitudes as determined from our CCD imaging.

14. and 15. Redshift and reference. Those prefixed ":" are from PKSCAT90 but are untraceable in the literature.

16. to 18. PKSCAT90 $S_{2.7 \mathrm{GHz}}, S_{5 \mathrm{GHz}}$ and $S_{\mathrm{lim}, 2.7 \mathrm{GHz}}$

19. Exclusion flag. If " $\mathrm{X}$ " then this source is excluded from our complete sample due to one of the following conditions: Source is non-compact radio source (NCS) as shown in Col. 8 and the individual notes for that source.

Source flux density at $2.7 \mathrm{GHz}$ is below the survey area flux density limit $\left(S_{2.7 \mathrm{GHz}}<S_{\lim , 2.7 \mathrm{GHz}}\right)$.

Source is steep-spectrum as described in detailed notes for that source.

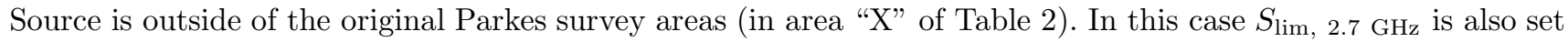
to 0.0 . 


\begin{tabular}{|c|c|c|c|c|c|c|c|c|c|c|c|c|c|c|c|c|c|}
\hline \multirow[b]{2}{*}{ Name } & \multicolumn{2}{|c|}{ PKSCAT90 B1950 } & \multicolumn{3}{|c|}{ New radio position $\mathrm{B} 1950$} & \multicolumn{5}{|c|}{ Offsets } & \multirow[b]{2}{*}{ Gunn- $i$} & \multirow[b]{2}{*}{ Gunn- $z$} & \multirow[b]{2}{*}{$z$} & \multirow[b]{2}{*}{$z \operatorname{Ref}$} & \multirow[b]{2}{*}{$S_{2.7}$} & \multirow[b]{2}{*}{$S_{5}$} & \multirow[b]{2}{*}{$S_{\lim }$} \\
\hline & $\mathrm{RA}$ & Dec & $\mathrm{RA}$ & Dec & & Ider & & RA & Dec & $B$ & & & & & & & \\
\hline PKS B0000-160 & 000053.7 & -160348 & 000053.68 & -160347.8 & $\mathrm{P}$ & 3 & $\mathrm{G}$ & -0.3 & -0.2 & 22.6 & 20.1 & 21.7 & 0.508 & 83 & 0.30 & 0.26 & 0.25 \\
\hline PKS B0001-121 & 000124.0 & -120636 & 000125.89 & -120616.2 & $\mathrm{P}$ & 3 & G & 2.6 & 1.0 & 24.0 & & & & & 0.27 & 0.22 & 0.25 \\
\hline PKS B0002-170 & 000243.6 & -170445 & 000244.52 & -170446.9 & $\mathrm{P}$ & $1 b$ & $\mathrm{Q}^{*}$ & -0.4 & -0.5 & 18.0 & & & & & 0.14 & 0.16 & $0.25 \mathrm{X}$ \\
\hline PKS B0002-478 & 000203.0 & -475302 & 000202.94 & -475302.1 & $\mathrm{C}$ & $1 \mathrm{a}$ & Q & & & 19.0 & & & & & 0.63 & 0.55 & 0.25 \\
\hline PKS B0003-066 & 000340.3 & $\begin{array}{lll}-06 & 4017\end{array}$ & 000340.29 & -064017.4 & $\mathrm{P}$ & $1 b$ & $\mathrm{BL}$ & -0.5 & -0.4 & 19.5 & & & 0.347 & 1 & 1.46 & 1.58 & 0.25 \\
\hline PKS B0005-239 & 000527.5 & -235600 & 000527.47 & -235600.1 & $\mathrm{P}$ & $1 b$ & $\mathrm{Q}$ & -0.2 & -0.8 & 17.0 & & & 1.410 & 2 & 0.58 & 0.53 & 0.25 \\
\hline PKS B0005-262 & 000553.5 & -261553 & 000553.48 & -261554.0 & $\mathrm{~N}$ & $1 \mathrm{a}$ & Q? & & & 20.0 & & & & & 0.58 & 0.58 & 0.25 \\
\hline PKS B0008-222 & 000821.1 & -221349 & 000821.03 & -221345.7 & $\mathrm{P}$ & 2 & G? & 0.6 & 0.5 & 21.8 & & 22.0 & & & 0.38 & 0.39 & 0.25 \\
\hline PKS B0008-264 & 000828.9 & -262915 & $00 \quad 0828.89$ & -262914.9 & $\mathrm{P}$ & $1 b$ & $\mathrm{Q}$ & -0.1 & 0.1 & 19.0 & & & 1.096 & 3 & 0.67 & 0.81 & 0.25 \\
\hline PKS B0008-307 & 000804.4 & -304413 & 000803.58 & -304429.1 & $\mathrm{~A}$ & 2 & Q & 0.2 & -0.5 & 19.1 & & & 1.189 & 83 & 0.31 & 0.27 & 0.20 \\
\hline PKS B0011-046 & 001120.6 & -044033 & 001120.66 & -044033.6 & $\mathrm{~N}$ & $1 \mathrm{a}$ & Q? & & & 19.0 & & & & & 0.21 & 0.41 & $0.25 \mathrm{X}$ \\
\hline PKS B0012-184 & 001230.0 & -182928 & 001230.06 & -182931.5 & $\mathrm{P}$ & $1 b$ & $\mathrm{Q}$ & 0.3 & -1.0 & 20.0 & & & 0.743 & 83 & 0.48 & 0.42 & 0.25 \\
\hline PKS B0013-005 & 001337.4 & -003153 & 001337.35 & -003152.6 & $\mathrm{P}$ & $1 b$ & Q & -0.1 & 0.1 & 19.5 & & & 1.575 & 4 & 0.80 & 0.65 & 0.10 \\
\hline PKS B0013-240 & 001333.8 & -240032 & 001333.87 & -240031.9 & $\mathrm{~N}$ & $1 \mathrm{a}$ & G & & & 17.0 & & & & & 0.25 & 0.23 & 0.25 \\
\hline PKS B0014-312 & 001455.6 & -311446 & & & & $1 \mathrm{a}$ & NCS & & & & & & & & 0.15 & 0.15 & $0.20 \mathrm{X}$ \\
\hline PKS B0017-307 & 001713.0 & -304756 & 001712.15 & -304759.0 & $\mathrm{~N}$ & $1 \mathrm{a}$ & $\mathrm{Q}$ & & & 19.0 & & & 2.666 & 83 & 0.34 & 0.33 & 0.20 \\
\hline PKS B0022-044 & 002212.8 & -042837 & 002212.74 & -042838.6 & $\mathrm{P}$ & $1 b$ & Q & -0.3 & 0.0 & 20.0 & & & 1.946 & 83 & 0.29 & 0.39 & 0.25 \\
\hline PKS B0026-014 & 002629.6 & -012942 & 002627.42 & -013016.7 & $\mathrm{P}$ & 2 & $\mathrm{G}$ & -0.9 & 0.5 & 16.3 & & & 0.083 & 83 & 0.30 & 0.26 & 0.25 \\
\hline PKS B0027-024 & 002758.9 & -022833 & 002758.41 & -022830.2 & $\mathrm{P}$ & 2 & $\mathrm{Q}^{*}$ & 1.1 & 0.5 & 19.6 & & & & & 0.22 & 0.18 & $0.25 \mathrm{X}$ \\
\hline PKS B0027-426 & 002751.8 & -424125 & & & & $1 \mathrm{a}$ & $\mathrm{Q}$ & & & 19.0 & & & 0.495 & 83 & 0.42 & 0.43 & 0.25 \\
\hline PKS B0028-396 & 002855.8 & -393931 & 002857.82 & -39 3923.2 & A & 2 & $\mathrm{Q}^{*}$ & -0.4 & -0.4 & 20.8 & & & & & 0.19 & 0.19 & $0.25 \mathrm{X}$ \\
\hline PKS B0029-414 & 002901.3 & -412439 & & & & $1 \mathrm{a}$ & $\mathrm{Q}$ & & & 17.0 & & & 0.896 & 5 & 0.23 & 0.21 & $0.25 \mathrm{X}$ \\
\hline PKS B0035-252 & 003546.0 & -251531 & 003546.01 & -251531.5 & $\mathrm{P}$ & $1 b$ & $\mathrm{Q}$ & -0.6 & -0.6 & 17.5 & & & 1.196 & 6 & 0.28 & 0.30 & 0.25 \\
\hline PKS B0035-595 & 003509.2 & -593427 & & & & $1 \mathrm{a}$ & $\mathrm{Q}$ & & & 19.0 & & & & & 0.26 & 0.21 & 0.25 \\
\hline PKS B0036-216 & 003600.4 & -213633 & 003600.45 & -213633.2 & $\mathrm{P}$ & $1 b$ & G & 0.0 & 0.2 & 19.0 & & & & & 0.53 & 0.60 & 0.25 \\
\hline PKS B0038-326 & 003805.1 & -324140 & 003804.09 & -324149.6 & $\mathrm{~S}$ & 2 & BL & 0.8 & 0.8 & 21.5 & & & & 83 & 0.43 & 0.69 & 0.20 \\
\hline PKS B0039-407 & 003937.6 & -404706 & & & & $1 \mathrm{a}$ & $\mathrm{Q}$ & & & 18.5 & & & 2.478 & 83 & 0.25 & 0.34 & 0.25 \\
\hline PKS B0040+001 & 004058.6 & +000838 & 004058.91 & +000834.3 & $\mathrm{~N}$ & $1 \mathrm{a}$ & Q & & & 19.0 & & & 1.127 & 7 & 0.08 & 0.11 & $0.10 \mathrm{X}$ \\
\hline PKS B0046-315 & 004658.3 & -313254 & 004657.68 & -313247.0 & $\mathrm{~N}$ & $1 \mathrm{a}$ & $\mathrm{Q}$ & & & 16.5 & & & 2.718 & 8 & 0.15 & 0.15 & $0.20 \mathrm{X}$ \\
\hline PKS B0047+023 & 004708.8 & +022043 & 004708.87 & +022043.0 & $\mathrm{~N}$ & $1 \mathrm{a}$ & $\mathrm{BL}$ & & & 18.0 & & & & 4 & 0.39 & 0.39 & 0.10 \\
\hline PKS B0047-051 & 004748.8 & -050839 & 004749.02 & -050840.0 & $\mathrm{P}$ & $1 b$ & $\mathrm{Q}^{*}$ & 0.1 & -0.2 & 18.5 & & & & & 0.18 & 0.27 & $0.25 \mathrm{X}$ \\
\hline PKS B0047-579 & 004748.0 & -575447 & 004748.16 & -575447.1 & $\mathrm{C}$ & $1 \mathrm{a}$ & $\mathrm{Q}$ & & & 17.8 & & & 1.797 & 8 & 1.96 & 2.48 & 0.25 \\
\hline PKS B0048-071 & 004836.2 & -070621 & 004836.22 & -070620.9 & $\mathrm{P}$ & $1 b$ & $\mathrm{Q}$ & -0.5 & 0.0 & 19.0 & & & 1.975 & 3 & 0.70 & 0.67 & 0.25 \\
\hline PKS B0048-097 & 004810.0 & -094524 & 004809.98 & -094524.3 & $\mathrm{P}$ & $1 b$ & $\mathrm{BL}$ & -0.4 & -0.2 & 17.0 & & & & 3 & 1.44 & 1.92 & 0.25 \\
\hline PKS B0048-427 & 004849.0 & -424252 & 004849.02 & -424252.0 & $\mathrm{C}$ & $1 \mathrm{a}$ & $\mathrm{Q}$ & & & 18.8 & & & 1.749 & 9 & 0.68 & 0.58 & 0.25 \\
\hline PKS B0051-077 & 005104.6 & -074345 & 005104.77 & -074345.9 & $\mathrm{P}$ & 3 & $\mathrm{G} ?$ & 0.6 & 0.2 & $>24.5$ & & 23.0 & & & 0.21 & 0.19 & $0.25 \mathrm{X}$ \\
\hline PKS B0054-006 & 005443.3 & -004046 & 005443.40 & -004045.7 & $\mathrm{P}$ & $1 b$ & $\mathrm{Q}$ & -0.5 & -0.2 & 18.0 & & & 2.790 & 8 & 0.15 & 0.19 & 0.10 \\
\hline PKS B0054-451 & 005429.0 & -450723 & & & & $1 \mathrm{a}$ & G & & & 18.5 & & & & & 0.46 & 0.39 & 0.25 \\
\hline PKS B0055-328 & 005542.0 & -325117 & & & & $1 \mathrm{a}$ & NCS & & & & & & & & 0.44 & 0.36 & $0.20 \mathrm{X}$ \\
\hline PKS B0056-525 & 005645.6 & -523520 & 005643.91 & -523536.9 & $\mathrm{~A}$ & 3 & $\mathrm{BL}$ & -1.2 & -1.6 & 23.6 & 20.9 & 23.5 & & 83 & 0.26 & 0.26 & 0.25 \\
\hline
\end{tabular}




\section{Appendix B: Notes on individual sources}

0000-160 Could be interacting with a fainter galaxy to south.

0001-121 Faint galaxy from CCD imaging, on $B$ frame only. However, the NVSS catalogues 2 bright radio sources, offset 87 and 297 arcsecs respectively with nothing closer to the Parkes or Patnaik position. The nearest NVSS radio source is coincident with a galaxy on DSS, with $B_{\mathrm{J}} \mathrm{mag}$ approx 21.0. It is probable that this is the correct radio source id, the Parkes beam being confused by 2 close compact radio sources. For the time being we leave the id as the faint galaxy and estimate the $B$ mag.

0005-262 PKSCAT90 galaxy is an incorrect identification: Identified as a stellar object on $K$-band image by Drinkwater et al. (1997).

0008-222 Classified as galaxy in the COSMOS survey, as probably extended on our Gunn-z image and also brighter on $B$.

0014-312 Undetected by our VLA observations, nothing in NVSS.

0026-014 A gE galaxy identified in the COSMOS survey, disturbed/interacting. Has smaller companion galaxy at same redshift.

0027-426 The redshift given in NED (from Hewitt et al. 1989) is wrong. New redshift obtained by us (Paper II).

0051-077 Not present on $B$ image. Classed as a galaxy as it is probably extended on Gunn-z image.

0055-328 Double radio source (NVSS). No compact core component at PKSCAT90 position.

0056-525 BL Lac object (Paper II), stellar on Gunn- $i$ and Gunn-z images, very faint on $B$.

0059-287 Re-observed with VLA 1.4 GHz April 95, showing steep radio spectrum; variable quasar?

0100-760 The correct redshift is given in Wilkes et al. (1983) and is not that of Jauncey et al. (1978).

0111-385 Galaxy identification from CCD frames. Our VLA observations indicate the source has a double radio structure; there is a candidate identification coincident with one component and is a faint diffuse red object. The NVSS survey also catalogues this as a double radio source and thus it is excluded from our complete sample.

0113-310 Original PKS survey catalogues this source as confused with 0112-310. NVSS catalogues this source 84 arcsec offset from the PKS position in RA: it is a compact radio source coincident with a stellar object.

0115-016 Extended (or compound) image appears on the Gunn- $i$ and Gunn-z, stellar on $B$.

0115-357 Nothing at position in NVSS survey, nearest NVSS source is 250 arcsec offset. Excluded from our complete sample.

0115-368 Extended, probably bent double radio source (NVSS). Excluded from our complete sample.

0123-016 = NGC 545/7; it is a very extended FRI radio source, and the apparent flat spectrum only comes about through inaccurate flux-density integration at 2.7 and 5.0 GHz. Excluded from our complete sample.

0128-132 Extended (bent double?) radio source (NVSS). Excluded from our complete sample.

0130-447 Low-surface-brightness galaxy identification from CCD imaging; red and very diffuse.

0143-061 Galaxy identified from the COSMOS survey with narrow-line spectrum (Paper II). Our VLA observations reveal an extended radio structure for the source, agreeing with NVSS. Excluded from our complete sample.

0144-219 Low-surface-brightness galaxy, most obvious on Gunn-i frame. Possibly interacting with nearby, brighter S0.

0156-144 Faint galaxy, from $B$ frame but not on Gunn-z. Galaxy is actually a steep-spectrum source: the PKSCAT90 2.7 GHz flux density is wrong (0.90 Jy vs. the original PKS survey value of 1.01 Jy). Our VLA observations reveal a steep-spectrum source, thus it is exluded from our complete sample.

0202-337 Scattered light from a nearby bright star creates a ghost ring covering the radio position, but the morphology is reasonably clear.

0203-520 Erroneous redshift given in PKSCAT90. Updated in our table to the correct value and reference.

0215+015 Extremely variable BL Lac object (Bolton \& Wall 1969; Gaskell 1982).

0215-015 Looks stellar on B and Z; no signal in our optical spectrum (Paper II) and thus unconfirmed quasar.

0218-683 Candidate is very close to bright star and its scattered light makes morphological classification difficult. 0225-065 The NVSS catalogues a compact source 3 arcsec from the PKSCAT90 position, at B1950 02 $25^{\mathrm{m}} 15.22$, $-06^{\circ} 34^{\prime} 32^{\prime \prime} .3,0.513 \mathrm{Jy}$ at $1.4 \mathrm{GHz}$. DSS shows a very faint object, possibly extended, close to this position; but it cannot be claimed as the identification.

0230-790 A suspect redshift: Browne \& Savage (1977) found a single line at $5800 \AA$ and it was pointed out by Jauncey et al. (2000) that if this were identified with MgII $\lambda 2798$ then the redshift is 1.07.

$\mathbf{0 2 3 1 + 0 2 2}$ Double radio source (NVSS), excluded from our complete sample.

0238-084 NGC 1052 LINER-type elliptical with inverted radio spectrum.

0239-553 Faint (possibly double) galaxy based on our ATCA position which notes it as a double radio source.

The centroid position is used for the identification. The relatively large radio-optical offset is probably due to the extended radio structure. Excluded from our complete sample.

0240-060 The redshift given in Strittmatter et al. (1974) is wrong. New redshift obtained by us (Paper II). 


\section{Appendix C: Finding charts for all sources}

Finding charts for all 878 sources presented in Appendix A. Charts are oriented North up, East left, and the scale is in arcmins. Position of the best radio position is marked $(+)$.

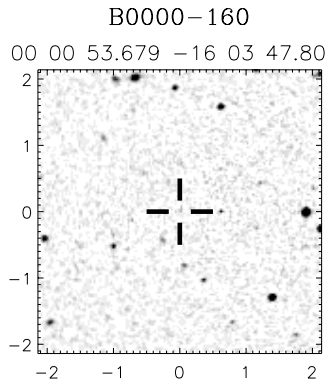

B0003-066

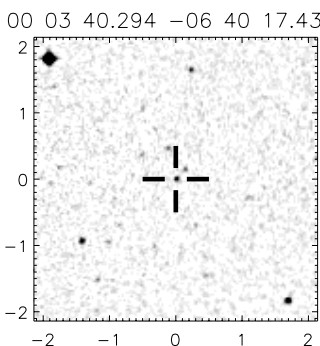

B0008-264

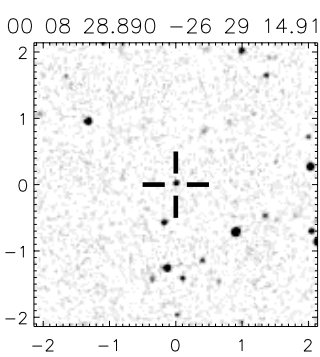

B0013-005

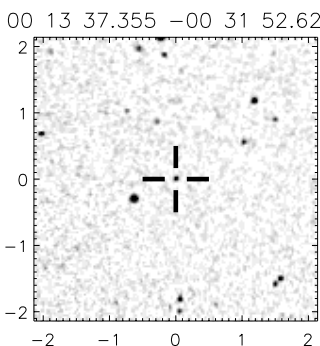

B0022-044

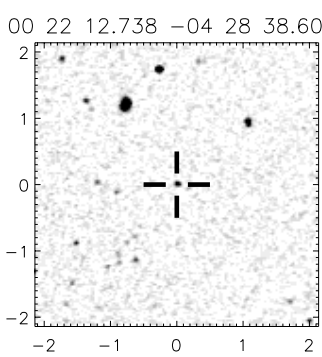

B0001-121

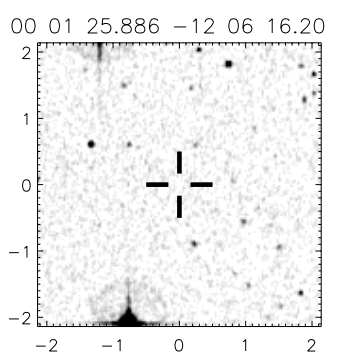

B0005-239

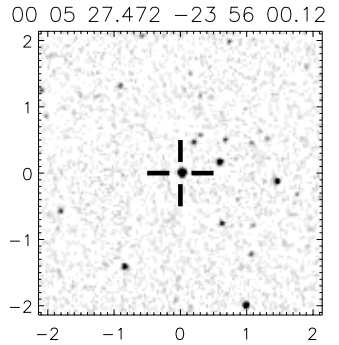

B0008-307

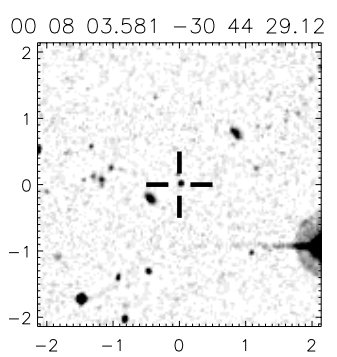

B0013-240

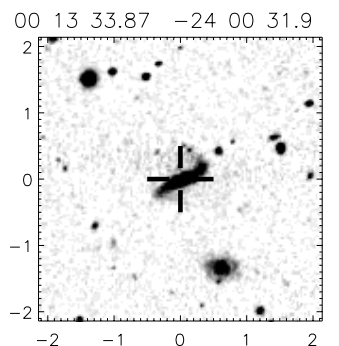

B0026-014

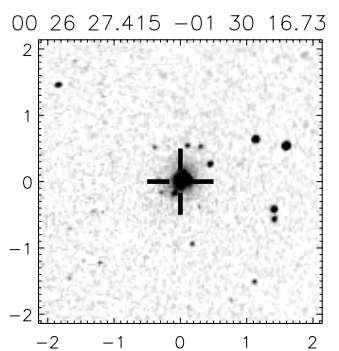

B0002-170

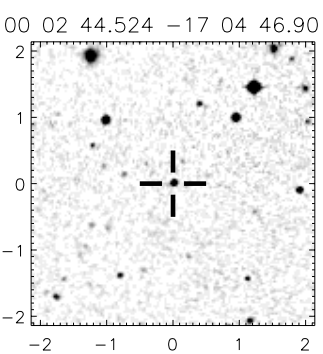

B0005-262

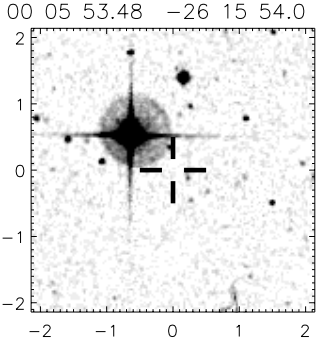

B0011-046

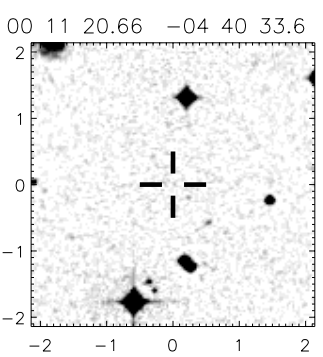

B0014-312

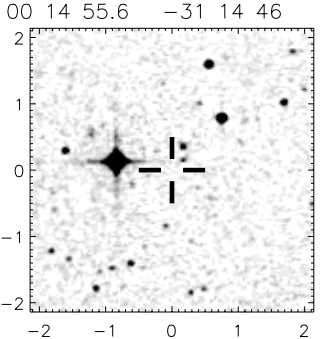

B0027-024

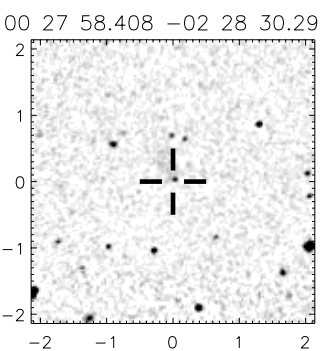

B0002-478

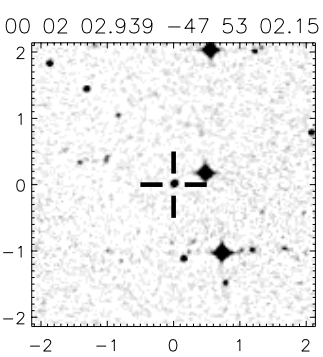

B0008-222

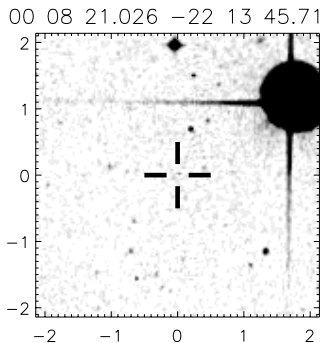

B0012-184

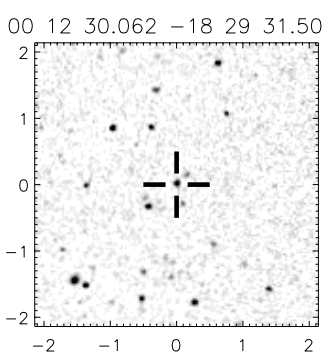

B0017-307

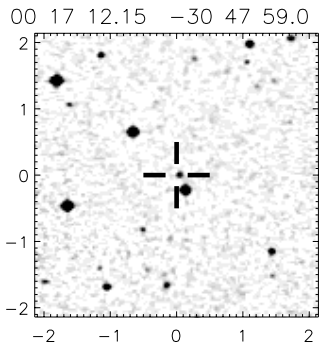

B0027-426

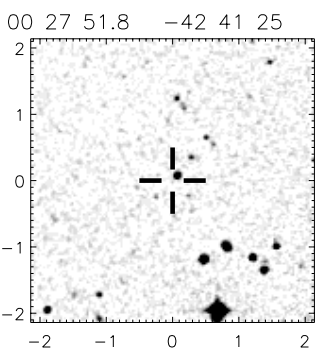




\section{Appendix D: Newly-identified sources with CCD images}

Images are oriented North up, East left, and scale is in arcseconds. Images are 2 arcminutes square, centered on the identification (o).
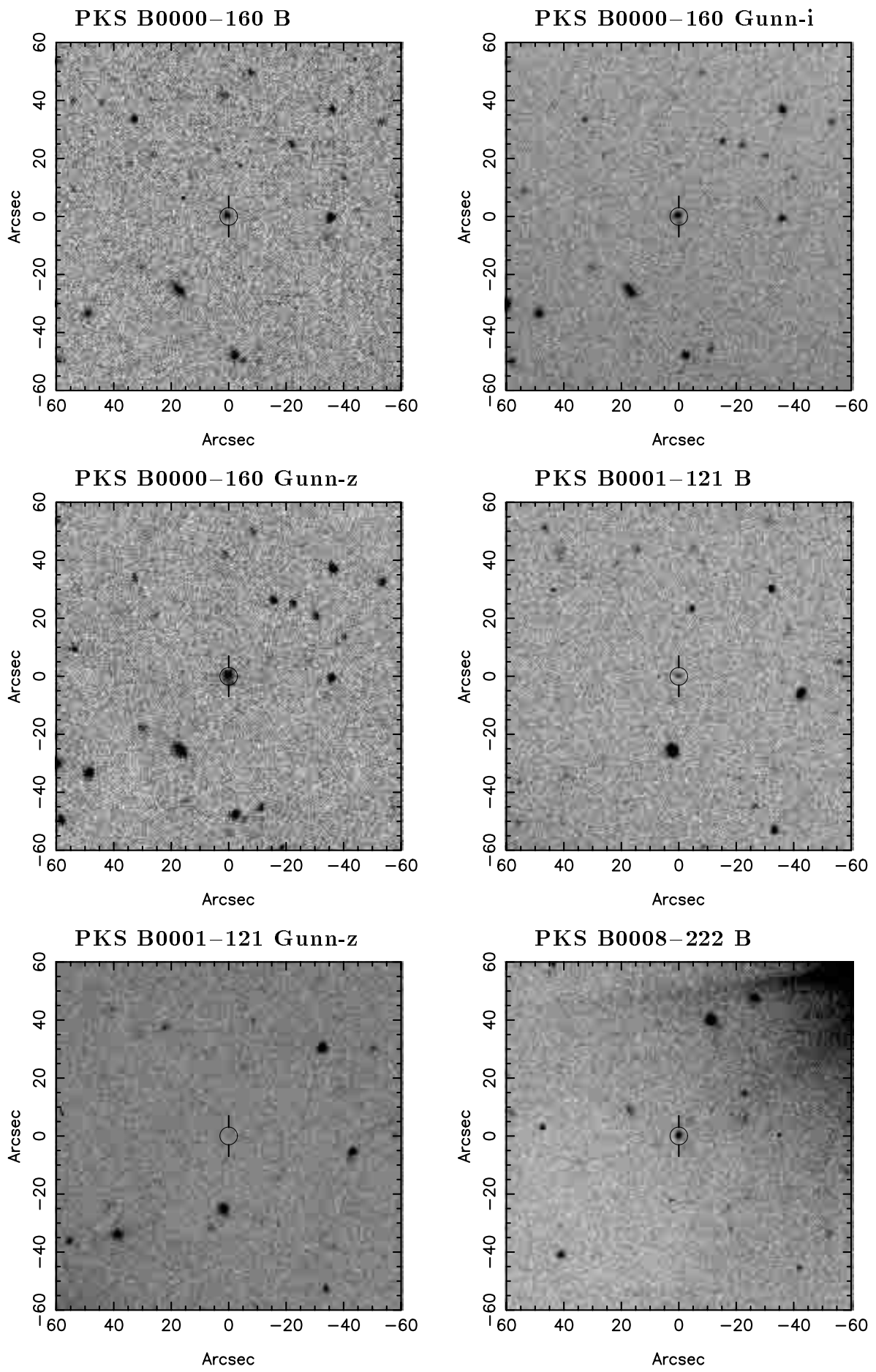


\section{References}

Abraham, R. G., Crawford, C. S., \& McHardy, I. M. 1991, MNRAS, 252, 482(35)

Allington-Smith, J. R., Peacock, J. A., \& Dunlop, J. S. 1991, MNRAS, 253, 287(19)

Baker, J. C., Hunstead, R. W., Kapahi, V. K., \& Subrahmanya, C. R. 1999, ApJS, 122, 29(55)

Baldwin, J. A., Wampler, E. J., \& Burbridge, E. M. 1981, ApJ, $243,76(63)$

Barbieri, C., Capaccioli, M., \& Zambon, M. 1975, Mem. Soc. Astron. Ital., 46, 461

Bergeron, J., \& Kunth, D. 1984, MNRAS, 207, 263(61)

Bock, D. C.-J., Large, M. I., \& Sadler, E. M. 1999, AJ, 117, 1578

Bolton, J. G., \& Butler, P. W. 1975, Aust. J. Phys. Astrophys. Suppl, 34, 33

Bolton, J. G., \& Ekers, J. A. 1966, Austr. J. Phys., 19, 275

Bolton, J. G., Kinman, T. D., \& Wall, J. V. 1968, ApJ, 154, L105(23)

Bolton, J. G., Savage, A., \& Wright, A. E. 1979, Aust. J. Phys. Suppl., 46, 1

Bolton, J. G., \& Shimmins, A. J. 1973, Aust. J. Phys. Astrophys. Suppl., 30, 1

Bolton, J. G., Shimmins, A. J., \& Wall, J. V. 1975, Australian J. Phys. Astrophys. Suppl., 34, 55

Bolton, J. G., \& Wall, J. V. 1969, Astrophys. Lett., 3, 177

Bolton, J., \& Wall, J. V. 1970, Aust. J. Phys., 23, 789

Bowyer, S., Brodie, J., Clarke, J. T., \& Henry, J. P. 1984, ApJ, 278, L103(77)

Browne, I. W. A., \& Savage, A. 1977, MNRAS, 179, 65P(22)

Browne, I. W. A., Savage, A., \& Bolton, J. G. 1975, MNRAS, $173,87 \mathrm{P}(12)$

Browne, I. W. A., \& Wright, A. E. 1985, MNRAS, 213, 97

Burbidge, E. M. 1970, ApJ, 160, L33(68)

Burbidge, E. M., \& Strittmatter, P. A. 1972a, ApJ, 172, L37(72)

Burbidge, E. M., \& Strittmatter, P. A. 1972b, ApJ, 174, L57(73)

Chu, Y. Q., Zhu, X. F., \& Butcher, H. 1986, Chinese Astron. Astrophys., 10, 196(28)

Condon, J. J., Cotton, W. D., Greisen, E. W., et al. 1998, AJ, 115,1693

Condon, J. J., Hicks, P. D., \& Jauncey, D. L. 1977, AJ, 82, 692

Condon, J. J., Jauncey, D. L., \& Wright, A. E. 1978, AJ, 83, 1036

Cristiani, S., \& Koehler, B. 1987, A\&AS, 68, 339(29)

da Costa, L. N., Willmer, C., Pellegrini, P. S., \& Chincarini, G. 1987, AJ, 93, 1338(62)

Danziger, I. J., \& Goss, W. M. 1979, MNRAS, 186, 93(71)

de Vaucouleurs, G., de Vaucouleurs, A., Corwin, H. G., et al. 1991, Reference Catalogue of Bright Galaxies, 3rd edition (Springer Verlag), 14

Dekker, H., \& D'Odorico, S. 1984, The Messenger, 37, 7(54)

di Serego-Alighieri, S., Danziger, I. J., Morganti, R., \& Tadhunter, C. N. 1994, MNRAS, 269, 998(65)

Downes, A. J. B., Peacock, J. A., Savage, A., \& Carrie, D. R. 1986, MNRAS, 218, 31

Dressler, A. 1991, ApJS, 75, 241(69)

Drinkwater, M. J., Barnes, D. G., \& Ellison, S. L. 1995, Proc. Astron. Soc. Aust., 12, 248

Drinkwater, M. J., Webster, R. L., Francis, P. J., et al. 1997, MNRAS, 284, 85(20)
Dunlop, J. S., Peacock, J. A., Savage, A., et al. 1989, MNRAS, $238,1171(4)$

Ekers, R. D., \& Ekers, J. A. 1973, A\&A, 24, 247

Eracleous, M., \& Halpern, J. P. 1994, ApJS, 90, 1(16)

Fairall, A. P., Willmer, C. N. A., Calderon, J. H., et al. 1992, AJ, 103, 11(59)

Falco, E. E., Kochanek, C. S., \& Munoz, J. A. 1998, ApJ, 494, 47

Falomo, R., Maraschi, L., Treves, A., \& Tanzi, E. G. 1987, ApJ, 318, L39(74)

Fisher, K. B., Huchra, J. P., Strauss, M. A., et al. 1995, ApJS, 100, 69(57)

Fricke, K. J., Kollatschny, W., \& Witzel, A. 1983, A\&A, 117, $60(24)$

Gaskell, C. M. 1982, ApJ, 252, 447

Goncalves, A. C., Veron, P., \& Veron-Cetty, M. P. 1998, A\&AS, $127,107(82)$

Heckman, T. M., O'Dea, C. P., Baum, S. A., \& Laurikainen, E. 1994, ApJ, 428, 65(39)

Hewitt, A., \& Burbidge, G. 1993, ApJS, 87, 451

Hewitt, P. C., Webster, R. L., Harding, M. E., et al. 1989, ApJ, 346, 1959

Hook, I. M., Shaver, P. A., Jackson, C. A., Wall, J. V., \& Kellermann, K. I. 2002, in preparation

Hunstead, R. W., \& Murdoch, H. S. 1980, MNRAS, 192, $31 \mathrm{P}(64)$

Hunstead, R. W., Murdoch, H. S., \& Shobbrook, R. R. 1978, MNRAS, 185, 149(26)

Jauncey, D. L., Batty, M. J., Gulkis, S., \& Savage, A. 1982, AJ, 87, 763(58)

Jauncey, D. L., Batty, M. J., Wright, A. E., Peterson, B. A., \& Savage, A. 1984, ApJ, 286, 498(6)

Jauncey, D. L., Kedziora-Chudczer, L. L., Macquart, J. P., et al. 2000, in IAU Symp., 205, E7

Jauncey, D. L., Savage, A., Morabito, D. D., et al. 1989, AJ, 98,54

Jauncey, D. L., Wright, A. E., Peterson, B. A., \& Condon, J. J. 1978, ApJ, 219, L1(5)

Junkkarinen, V. 1984, PASP, 96, 539(66)

Kollgaard, R. I., Feigelson, E. D., Laurent-Muehleisen, S. A., et al. 1995, ApJ, 449, 61

Landolt, A. U. 1992, AJ, 104, 340

Lanzetta, K. M., McMahon, R. G., Wolfe, A. M., et al. 1991, ApJS, 77, 1(70)

Lynds, C. R. 1967, ApJ, 147, 837(21)

Lynds, C. R., Hill, S. J., Heere, K., \& Stockton, A. N. 1966 ApJ, 144, 1244(51)

Lynds, C. R., Stockton, A. N., \& Livingston, W. C. 1965, ApJ, $142,1667(53)$

Maza, J., Ruiz, M. T., Gonzalez, L. E., Wischnjewsky, M., \& Antezana, R. 1993, Rev. Mex. Astron. Astrofis., 25, 51(79)

McAlpine, G. M., \& Feldman, F. R. 1982, ApJ, 261, 412(13)

Morton, D. C., Savage, A., \& Bolton, J. G. 1978, MNRAS, 185, $735(36)$

Murdoch, H. S., Hunstead, R. W., Blades, J. C., \& Pettini, M. 1986, Ap\&SS, 118, 501(18)

O’Dea, C. P., Baum, S. A., Stanghellini, C., et al. 1990, A\&AS, 84,549

Osmer, P. S., \& Smith, M. G. 1980, ApJS, 42, 333(80)

Owen, F. N., Ledlow, M. J., \& Keel, W. C. 1995, AJ, 109, 14(44)

Perlman, E. S., Padovani, P., Giommi, P., et al. 1998, AJ, 115, 1253(37) 
Peterson, B. A., \& Bolton, J. G. 1972, ApJ, 173, L19(25)

Peterson, B. A., Jauncey, D. L., Wright, A. E., \& Condon, J. J. 1976, ApJ, 207, L5(8)

Peterson, B. A., Wright, A. E., Jauncey, D. L., \& Condon, J. J. 1979, ApJ, 232, 400(45)

Postman, M., \& Lauer, T. R. 1995, ApJ, 440, 28(56)

Quintana, H., \& Ramirez, A. 1995, ApJS, 96, 343(43)

Reimers, D., Koehler, T., \& Wisotzki, L. 1996, A\&AS, 115, 235(48)

Roeser, S., Bastian, U., \& Kuzmin, A. 1994, A\&AS, 105, 301

Savage, A., \& Bolton, J. G. 1979, MNRAS, 188, 599(17)

Savage, A., Bolton, J. G., \& Trett, J. 1982, Aust. J. Phys., 35, 207

Savage, A., Browne, I. W. A., \& Bolton, J. G. 1976, MNRAS, $177,77 \mathrm{P}(30)$

Savage, A., Clowes, R. G., Cannon, R. D., et al. 1985, MNRAS, $213,485(11)$

Savage, A., \& Wright, A. E. 1981, MNRAS, 196, 927(75)

Savage, A., Wright, A. E., \& Bolton, J. G. 1977, Aust. J. Phys. Astrophys. Suppl, 4, 21

Schmidt, M. 1963, Nature, 197, 1040(52)

Schmidt, M. 1977, ApJ, 217, 358(34)

SDSS 2001, Sloan Digital Sky Survey (early-release data), http://archive.stsci.edu/sdss/

Searle, L., \& Bolton, J. G. 1968, ApJ, 154, L101(76)

Shaver, P. A., Wall, J. V., Kellermann, K. I., Jackson, C. A., \& Hawkins, M. R. S. 1996, Nature, 384, 439

Shimmins, A. J. 1971, Aust. J. Phys. Astrophys. Suppl., 21, 1

Shimmins, A. J., \& Bolton, J. G. 1972, Aust. J. Phys. Astrophys. Suppl., 26, 1

Shimmins, A. J., \& Bolton, J. G. 1974, Aust. J. Phys. Astrophys. Suppl., 32, 1

Smith, A. G., \& Nair, A. D. 1995, PASP, 107, 863

Stanghellini, C., Baum, S. A., O'Dea, C. P., \& Morris, G. B. 1990, A\&A, 233, 379

Stickel, M., Fried, J. W., \& Kuehr, H. 1993a, A\&AS, 98, 393(78)

Stickel, M., Fried, J. W., \& Kuehr, H. 1989, A\&AS, 80, 103(1)

Stickel, M., Kuehr, H., \& Fried, J. W. 1993b, A\&AS, 97, 483(38)

Stickel, M., \& Kuhr, H. 1993, A\&AS, 101, 521(41)

Stobie, R. S., Sagar, R., \& Gilmore, G. 1985, A\&AS, 60, 503

Strittmatter, P. A., Carswell, R. F., \& Gilbert, G. 1974, ApJ, 190, 509(27)

Tadhunter, C. N., Morganti, R., di Serego-Alighieri, S., Fosbury, R. A. E., \& Danziger, I. J. 1993, MNRAS, 263, 999(42)

Thompson, D. J., Djorgovski, S., \& de Carvalho, R. 1990, PASP, 102, 1235
Veron, P., Veron-Cetty, M. P., Djorgovski, S., et al. 1990, A\&AS, 86, 543(32)

Veron-Cetty, M. P., \& Veron, P. 1989, QSO list (ESO Scientific Report, Garching: European Southern Observatory (ESO), 1989, 4th ed.)

Veron-Cetty, M.-P., \& Veron, P. 1993, A\&AS, 100, 521

Veron-Cetty, M. P., \& Veron, P. 1998, A Catalogue of quasars and active nuclei (A Catalogue of quasars and active nuclei, Edition: 8th ed., Publisher: Garching: European Southern Observatory (ESO), 1998, Ser. ESO Scientific Report Ser. Vol. 18)

Wall, J. V., \& Peacock, J. A. 1985, MNRAS, 216, 273

Wall, J. V., Shaver, P. A., Jackson, C. A., Kellermann, K. I., \& Hook, I. M. 2002, in preparation

Wall, J. V., Shimmins, A. J., \& Bolton, J. G. 1975, Aust. J. Phys. Astrophys. Suppl., 34, 55

Wall, J. V., Shimmins, A. J., \& Merkelijn, J. K. 1971, Aust. J. Phys. Suppl., 19, 1

Wall, J. V., Wright, A. E., \& Bolton, J. G. 1976, Aust. J. Phys. Astrophys. Suppl., 39, 1

White, G. L. 1992, Proc. Astron. Soc. Aust., 10, 140

White, G. L., Jauncey, D. L., Savage, A., et al. 1988, ApJ, 327, $561(9)$

Wilkes, B. 1986, MNRAS, 218, 331(31)

Wilkes, B. J., Wright, A. E., Jauncey, D. L., \& Peterson, B. A. 1983, Proc. Astron. Soc. Aust., 5, 2(10)

Wills, B. J., \& Wills, D. 1979, ApJS, 41, 689(81)

Wills, D., \& Lynds, R. 1978, ApJS, 36, 317(7)

Wills, D., \& Wills, B. J. 1976, ApJS, 31, 143(2)

Wright, A. E., Ables, J. G., \& Allen, D. A. 1983, MNRAS, 205, $793(3)$

Wright, A. E., Jauncey, D. L., Bolton, J. G., \& Savage, A. 1982, Aust. J. Phys., 35, 177

Wright, A. E., Jauncey, D. L., Peterson, B. A., \& Condon, J. J. 1977, ApJ, 211, L115(15)

Wright, A. E., \& Otrupcek, R. E. 1990, Parkes Catalogue, Australia Telescope National Facility, CSIRO

Wright, A. E., Peterson, B. A., Jauncey, D. L., \& Condon, J. J. 1978, ApJ, 226, L61(40)

Wright, A. E., Peterson, B. A., Jauncey, D. L., \& Condon, J. J. 1979, ApJ, 229, 73(33)

Yentis, D. J., Cruddace, R. G., Gursky, H., et al. 1992, in Digitized Optical Sky Surveys, ed. H. T. McGillivray, \& E. B. Thomson (Kluwer, Dordrecht)

Young, P., Sargent, W. L. W., \& Boksenberg, A. 1982, ApJS, 48, 455(49)

Zickgraf, F. J., Voges, W., Krautter, J., et al. 1997, A\&A, 323, L21(47) 